\title{
An Analytical Derivation of Ice-Shelf Basal Melt Based on the Dynamics of Meltwater Plumes
}

\author{
WERNER M. J. LAZEROMS \\ Institute for Marine and Atmospheric Research Utrecht, Utrecht University, Utrecht, Netherlands
}

ADRIAN JENKINS

British Antarctic Survey, Natural Environment Research Council, Cambridge, United Kingdom

SJOERD W. RIENSTRA

Department of Mathematics and Computer Science, Eindhoven University of Technology, Eindhoven, Netherlands

\author{
RODERIK S. W. VAN DE WAL
}

Institute for Marine and Atmospheric Research Utrecht, and Faculty of Geosciences, Department of Physical Geography, Utrecht University, Utrecht, Netherlands

(Manuscript received 20 June 2018, in final form 30 November 2018)

\begin{abstract}
The interaction between ice shelves and the ocean is an important process for the development of marine ice sheets. However, it is difficult to model in full detail due to the high computational cost of coupled iceocean simulations, so that simplified basal-melt parameterizations are required. In this work, a new analytical expression for basal melt is derived from the theory of buoyant meltwater plumes moving upward under the ice shelf and driving the overturning circulation within the ice-shelf cavity. The governing equations are nondimensionalized in the case of an ice shelf with constant basal slope and uniform ambient ocean conditions. An asymptotic analysis of these equations in terms of small slopes and small thermal driving, assumed typical for Antarctic ice shelves, leads to an equation that can be solved analytically for the dimensionless melt rate. This analytical expression describes a universal melt-rate curve onto which the scaled results of the original plume model collapse. Its key features are a positive melt peak close to the grounding line and a transition to refreezing further away. Comparing the analytical expression with numerical solutions of the plume model generally shows a close agreement between the two, even for more general cases than the idealized geometry considered in the derivation. The results show how the melt rates adapt naturally to changes in the geometry and ambient ocean temperature. The new expression can readily be used for improving ice-sheet models that currently still lack a sufficiently realistic description of basal melt.
\end{abstract}

\section{Introduction}

The interaction between marine ice sheets and the surrounding ocean currents has received increased attention in recent years due to its potential importance for the overall mass balance of ice sheets and an associated rapid sea level rise. The system in which these interactions occur can take on the form of tidewater glaciers with a near-vertical edge terminating in the ocean or floating ice shelves attached to the grounded

Corresponding author: Roderik S. W. van de Wal, r.s.w.vandewal@ uu.nl ice sheet. The latter case is especially important for the Antarctic Ice Sheet (AIS), which is buttressed by a vast number of floating ice shelves along its margin. As shown in recent studies (Pritchard et al. 2012; Depoorter et al. 2013; Rignot et al. 2013; Golledge et al. 2015; DeConto and Pollard 2016), subshelf basal melting is a major factor in the mass loss of the AIS and its potential contribution to future sea level rise, particularly in the warmer waters of the Amundsen Sea sector (Rignot et al. 2014).

These aspects demonstrate the need for accurate models of the interaction between ice shelves and ocean. Traditionally, the main mechanism behind this 
interaction has been described by a buoyancy-driven overturning circulation beneath the floating ice shelf (MacAyeal 1985; Hellmer and Olbers 1989; Jenkins 1991). Fresh meltwater, generated either directly at the ice-ocean interface or at the grounding line in the form of subglacial discharge, is positively buoyant in the saline environment of the ice-shelf cavity and moves upward under the ice shelf base, creating a turbulent plume. Due to entrainment of the ambient water within the cavity or the possible inflow of warmer ocean waters, the plume can generate more basal melt. In stratified environments, the plume can also detach from the ice-shelf base when reaching a level of neutral buoyancy, leading to more complicated circulation patterns and different melting modes (Jacobs et al. 1992). However, applying these notions in the context of large-scale climate simulations with ice-sheet models and/or ocean general circulation models remains problematic (Asay-Davis et al. 2017).

From an ocean-modeling perspective, basal melt rates can be calculated by explicitly resolving the cavity circulation, using a parameterization of heat exchange to the ice shelf. This is currently only feasible in sufficient detail for single ice-shelf cavities for which the geometry is well known (e.g., Thoma et al. 2015; Asay-Davis et al. 2016; De Rydt and Gudmundsson 2016; Seroussi et al. 2017; Timmermann and Goeller 2017). Several experiments have been done with high-resolution models on continental scale to simulate the subshelf circulation and basal melt rates (e.g., Timmermann and Hellmer 2013; Dinniman et al. 2015; Mathiot et al. 2017; Naughten et al. 2018), but they are computationally expensive, especially if coupled to a dynamical ice-sheet model that captures geometry changes over long time scales. From an icemodeling perspective with standalone ice-sheet models (e.g., De Boer et al. 2015), basal melt is usually described by highly simplified expressions based on the local iceocean flux (Beckmann and Goosse 2003), which by themselves do not explicitly account for the cavity circulations. The feedback between basal melt and the cavity circulation can be partially captured by a simple quadratic temperature dependence, as described by Holland et al. (2008) and applied by DeConto and Pollard (2016), which, however, still lacks important geometry-dependent effects.

Instead of fully resolving the ice-shelf cavity circulation, it would have great computational advantages if its dynamical features could be included in a straightforward way in a basal melt parameterization, which is the aim of this paper. The starting point is the quasione-dimensional plume model by Jenkins (1991) that, although simplified, describes the basic physics of the aforementioned buoyant meltwater plumes driving the cavity circulation. Jenkins (2011) already showed how under certain conditions the results of this plume model scale to a rather universal relation for the basal melt rates caused by subglacial discharge at the grounding line. However, it was shown that the length scale over which melting is directly influenced by this freshwater input at the grounding line (convection-driven melting) is typically small for ice shelves. Beyond this small distance from the grounding line, the dominant mechanism for plume dynamics is caused by the basal melt itself (meltdriven convection). A governing length scale for this mechanism was found by Lane-Serff (1995), depending on the ambient ocean temperature and the local freezing point. This regime, in which the depth-dependent freezing point dominates the cavity circulation, is central in the current study. It should be noted, however, that other processes remain important for basal melting of ice shelves, including tidal forcing (e.g., Mueller et al. 2012), the aforementioned subglacial discharge (e.g., Jenkins 2011; Slater et al. 2017), stratification of the ambient cavity water (e.g., Magorrian and Wells 2016), and the impact of frazil ice formations on the plume dynamics (e.g., Smedsrud and Jenkins 2004).

To obtain a practical parameterization describing subshelf basal melt rates in the dominant regime, Jenkins (2014) performed a second empirical analysis of the plume model results, again leading to a universal scaling for the melt rate that extends the analysis of Lane-Serff (1995). This parameterization unites the influence of geometry (basal slope and depth) with the dependence on ambient ocean properties. A key feature of the parameterization is a dimensionless melt-rate curve that contains a positive peak near the grounding line and a transition to refreezing further away. Lazeroms et al. (2018) applied the new parameterization to all Antarctic ice shelves and showed an improvement in modeled melt rates compared with simpler parameterizations (e.g., Beckmann and Goosse 2003), especially in terms of spatial variations and temperature sensitivity. By parameterizing the melt rates using the dynamical features of the plume model, one implicitly accounts for important effects of the cavity circulation without explicitly resolving it. Note that an alternative approach with similar behavior exists in the form of the box model by Olbers and Hellmer (2010), which was successfully applied to Antarctic ice shelves by Reese et al. (2018).

However, the empirical melt-rate curve used in Lazeroms et al. (2018) does not immediately provide insight in the underlying physical assumptions from which it was derived. It is essentially a polynomial fit of the scaled numerical data from the plume model. Lazeroms et al. (2018) provided a quick calculation from simplified equations that could retrieve the correct 


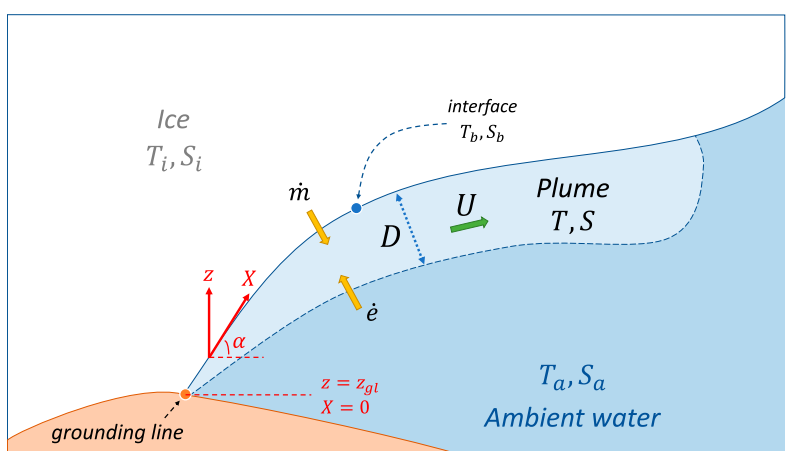

FIG. 1. Schematic picture of the plume model of Jenkins $(1991,2011)$ for a typical ice-shelf geometry with basal slope $\alpha(X)$. The plume is described by a thickness $D$, speed $U$, temperature $T$, and salinity $S$ and forced by entrainment $\dot{e}$ of ambient ocean water and basal melting $\dot{m}$.

scaling factors, but not the dimensionless melt-rate curve itself. Moreover, a major practical drawback of this curve is its formulation in terms of a polynomial of degree 11, which is very sensitive to the numerical values of its coefficients and prone to implementation errors. Therefore, a more robust formulation in terms of a systematically derived analytical expression is desirable.

In this paper, we attempt to formalize the empirical analysis and the resulting basal-melt parameterization of Jenkins (2014). Starting with the plume model by Jenkins (1991), we show how an appropriate scaling of the plume model equations leads to a dimensionless system that can be solved analytically under certain conditions. This yields an analytical expression for the basal melt rate that is nearly identical to the empirical melt-rate curve of Jenkins (2014) and allows simple use in ice-sheet models. Knowing how these expressions are derived systematically from the underlying physical equations is important for understanding both the potential and the drawbacks of the parameterization for use in ice-sheet models, and it sheds light on a possible extension including more complex physics.

In the next section, we present the derivation of the basal melt rate starting with a brief description of the underlying plume model. The plume model equations are simplified step by step, by first assuming a constant basal slope and uniform ambient ocean properties and then applying an asymptotic analysis for small values of the slope and the thermal driving of the plume, which we show to be typical for (Antarctic) ice shelves. In section 3, we show numerical results comparing the analytical expression with the full plume model for various cases both within and beyond the assumptions of the formal derivation. Section 4 provides concluding remarks and a brief discussion of the remaining practical issues for numerical ice-sheet models.

\section{Derivation of the model}

In this section we derive the new analytical expression for the basal-melt rate beneath a floating ice shelf, based on the plume model by Jenkins $(1991,2011)$. Although the original model is designed for a general quasi-onedimensional geometry of the ice-shelf base and general temperature and salinity profiles for the ambient ocean water, we will assume a constant basal slope and constant ambient properties to simplify the analysis. Using a suitable scaling and perturbation methods, this analysis leads to a dimensionless melt-rate curve that can be applied in more general cases, as shown in section 3 .

\section{a. Plume model equations}

The plume model of Jenkins $(1991,2011)$ describes the evolution of a buoyant meltwater plume beneath an ice shelf with a basal geometry that is uniform in the cross-flow direction (Fig. 1). This quasi-one-dimensional geometry can be described by a slope angle $\alpha$ that essentially depends on the basal depth $z_{b}$ or, equivalently, the alongslope coordinate $X$, where $X=0$ corresponds to the grounding-line depth $z_{\mathrm{gl}}$. Note that all $z$ values are defined as increasing upward with zero position at sea level. Furthermore, the ambient ocean water in the ice-shelf cavity has temperature $T_{a}$ and salinity $S_{a}$, which in principle can be depth dependent as well.

The dynamics of the plume under the ice shelf in Fig. 1 can be modeled by a two-layer system, in which the plume thickness $D$ and the (depth-averaged) plume velocity $U$, plume temperature $T$, and plume salinity $S$ as functions of $X$ are described by the following system:

$$
\begin{aligned}
\frac{d D U}{d X} & =\dot{e}+\dot{m} \\
\frac{d D U^{2}}{d X} & =D \frac{\Delta \rho}{\rho_{0}} g \sin \alpha-C_{d} U^{2},
\end{aligned}
$$

$$
\begin{aligned}
& \frac{d D U T}{d X}=\dot{e} T_{a}+\dot{m} T_{b}-C_{d}^{1 / 2} \Gamma_{T} U\left(T-T_{b}\right), \quad \text { and } \\
& \frac{d D U S}{d X}=\dot{e} S_{a}+\dot{m} S_{b}-C_{d}^{1 / 2} \Gamma_{S} U\left(S-S_{b}\right),
\end{aligned}
$$

corresponding to conservation of mass, momentum, heat, and salt, respectively. The first equation describes the plume volume flux $D U$ as being determined by the melt rate $\dot{m}$ (input of meltwater from the ice-ocean interface) and the entrainment rate $\dot{e}$ (input of ambient ocean water), which in turn is modeled by (Bo Pedersen 1980):

$$
\dot{e}=E_{0} U \sin \alpha,
$$


where $E_{0}$ is a dimensionless constant. As already explained by Jenkins (1991), this entrainment parameterization is chosen for its simple dependence on plume velocity and basal slope, giving good agreements with laboratory studies for small slopes, but we note that more general 2D parameterizations without an explicit slope dependence exist (see, e.g., Sergienko 2013).

The second equation [(1b)] provides the balance between the driving force (buoyancy), determined by the density contrast $\Delta \rho=\rho_{a}-\rho$ of the plume with respect to the ambient ocean, and the drag force with dimensionless drag coefficient $C_{d}$. The density contrast is found from a linearized equation of state:

$$
\frac{\Delta \rho}{\rho_{0}}=\beta_{S}\left(S_{a}-S\right)-\beta_{T}\left(T_{a}-T\right),
$$

where $\beta_{S}$ is the haline contraction coefficient and $\beta_{T}$ the thermal expansion coefficient. Note that Coriolis forces have been neglected in (1b), as well as along-stream variations in the buoyancy $d(D \Delta \rho) / d X$ (see, e.g., Sergienko 2013). Coriolis forces are known to have a significant effect on larger ice shelves, but Jenkins (2011) provides an estimate of rotational length scales, on which we will briefly comment in section 3c. A more detailed analysis of the momentum equation was performed by Mahrt (1982), showing that the along-stream variations in buoyancy can generally be neglected. This assumption most notably breaks down on the outer parts of ice shelves, where the plume decelerates due to either a loss of buoyancy or a reduction in the basal slope.

Equations (1c) and (1d) describe the input of heat and salt due to entrainment $\dot{e}$ and melting $\dot{m}$, as well as turbulent exchange through the sub-ice-shelf boundary layer with exchange coefficients (Stanton numbers) $C_{d}^{1 / 2} \Gamma_{T}$ and $C_{d}^{1 / 2} \Gamma_{S}$. Furthermore, $T_{b}$ and $S_{b}$ are the temperature and salinity at the ice-ocean interface. To close the system, we require three additional conditions at the ice-ocean interface (Jenkins 1991, 2011):

$$
\begin{aligned}
C_{d}^{1 / 2} \Gamma_{T} U\left(T-T_{b}\right) & =\dot{m}\left[\frac{L}{c}+\frac{c_{i}}{c}\left(T_{b}-T_{i}\right)\right], \\
C_{d}^{1 / 2} \Gamma_{S} U\left(S-S_{b}\right) & =\dot{m}\left(S_{b}-S_{i}\right), \quad \text { and } \\
T_{b} & =\lambda_{1} S_{b}+\lambda_{2}+\lambda_{3} z_{b} .
\end{aligned}
$$

Equations (4a) and (4b) describe the balance of heat and salt fluxes at the interface, where $T_{i}$ and $S_{i}$ are the temperature and salinity of the ice, $L$ is the latent heat of fusion for ice, and $c_{i}$ and $c$ are the specific heat capacities of ice and ocean water, respectively. Finally, (4c) equates the interface temperature $T_{b}$ with the local pressure freezing point for salinity $S_{b}$ at depth $z_{b}$, given by the (linearized) liquidus condition with constant coefficients $\lambda_{1}, \lambda_{2}$, and $\lambda_{3}$.

The plume model in the form presented above can be solved numerically for any quasi-one-dimensional ice-shelf geometry, defined by the draft $z_{b}(X)$ and slope angle $\alpha(X)$, and any vertical profile for the ambient ocean properties $T_{a}(z)$ and $S_{a}(z)$. The constant parameter values used in this study are summarized in Table 1. Furthermore, the plume model requires four initial conditions at the grounding line $(X=0)$ for the quantities $D, U, T$, and $S$ [(1)]. In general, one can impose an initial freshwater flux $(D U)_{X=0}$ at the grounding line with zero salinity and temperature equal to the local pressure freezing point, as discussed in Jenkins (2011). However, in contrast to the aforementioned paper, we are mainly interested in the regime of melt-driven convection where the primary source of buoyancy is the meltwater generated locally at the ice-shelf base. Therefore, we take $D=U=0$ as initial condition at the grounding line throughout this paper. The numerical solution of the plume model will be revisited in section 3 , where it is compared with the analytical formulation derived in the following.

\section{b. Simplified formulation and scaling}

To facilitate the analytical derivation, we shall apply a number of simplifications to the original plume model. First of all, following Jenkins (2011), the interface conditions in (4) can be replaced by a simpler formulation (McPhee 1992; McPhee et al. 1999) consisting of only two equations:

$$
\begin{aligned}
C_{d}^{1 / 2} \Gamma_{T S} U\left(T-T_{f}\right) & =\dot{m}\left[\frac{L}{c}+\frac{c_{i}}{c}\left(T_{f}-T_{i}\right)\right], \quad \text { and } \\
T_{f} & =\lambda_{1} S+\lambda_{2}+\lambda_{3} z_{b}
\end{aligned}
$$

where $T_{f}$ is the pressure freezing point of the plume, taking over the role of the interface temperature $T_{b}$, and $C_{d}^{1 / 2} \Gamma_{T S}$ is an effective heat exchange coefficient. This simplification also requires replacing $T_{b}$ by $T_{f}$ and $\Gamma_{T}$ by $\Gamma_{T S}$ in the plume heat balance in (1c). Note that the interface salinity $S_{b}$ can be eliminated directly by substituting (4b) in the salinity equation (1d), where the ice salinity $S_{i}$ is assumed to be zero. Furthermore, the ice temperature $T_{i}$ is eliminated by neglecting the conductive heat flux [the last term on the right-hand side in (5a)], considering that $c_{i}\left(T_{f}-T_{i}\right) \ll L$. Alternatively, one could assume the heat conduction term to be constant, as in Jenkins (2011), which would not change the algebra because this term can be incorporated as a small correction to the value of $L$. Compared to the three-equation formulation in (4), the two-equation formulation in (5) can 
TABLE 1. Constant parameters used in the plume model (Jenkins 1991, 2011) and the (derivation of the) melt-rate parameterization (28). The values for $c, \beta_{S}, \beta_{T}, \lambda_{1}, \lambda_{2}$, and $\lambda_{3}$ are for potential temperature and practical salinity units. Note that the values of $c_{\rho 1}, c_{\rho 2}$, and $c_{\tau}$ technically depend on the ambient salinity $S_{a}$; the presented values are for $S_{a}=34.65$ psu.

\begin{tabular}{|c|c|c|}
\hline & Plume model parameters & Values \\
\hline$E_{0}$ & Entrainment coefficient & $3.6 \times 10^{-2}$ \\
\hline$C_{d}$ & Drag coefficient & $2.5 \times 10^{-3}$ \\
\hline$\lambda_{1}$ & $\begin{array}{l}\text { Freezing point salinity } \\
\text { coefficient }\end{array}$ & $-5.73 \times 10^{-2}{ }^{\circ} \mathrm{C}$ \\
\hline$\lambda_{2}$ & Freezing point offset & $8.32 \times 10^{-2}{ }^{\circ} \mathrm{C}$ \\
\hline$\lambda_{3}$ & Freezing point depth coefficient & $7.61 \times 10^{-4}{ }^{\circ} \mathrm{Cm}^{-1}$ \\
\hline$C_{d}^{1 / 2} \Gamma_{T}$ & Thermal Stanton number & $1.1 \times 10^{-3}$ \\
\hline$C_{d}^{1 / 2} \Gamma_{S}$ & Haline Stanton number & $3.1 \times 10^{-5}$ \\
\hline$L^{a}$ & Latent heat of fusion for ice & $3.35 \times 10^{5} \mathrm{~J} \mathrm{~kg}^{-1}$ \\
\hline$c$ & $\begin{array}{l}\text { Specific heat capacity of ocean } \\
\text { water }\end{array}$ & $3.974 \times 10^{3} \mathrm{~J} \mathrm{~kg}^{-1}{ }^{\circ} \mathrm{C}^{-1}$ \\
\hline$c_{i}$ & Specific heat capacity of ice & $2.009 \times 10^{3} \mathrm{~J} \mathrm{~kg}^{-1}{ }^{\circ} \mathrm{C}^{-1}$ \\
\hline$\beta_{S}$ & Haline contraction coefficient & $7.86 \times 10^{-4} \mathrm{psu}^{-1}$ \\
\hline$\beta_{T}$ & Thermal expansion coefficient & $3.87 \times 10^{-5}{ }^{\circ} \mathrm{C}^{-1}$ \\
\hline$g$ & Gravitational acceleration & $9.81 \mathrm{~m} \mathrm{~s}^{-2}$ \\
\hline \multicolumn{2}{|c|}{ Melt-rate parameterization } & Values \\
\hline$C_{d}^{1 / 2} \Gamma_{T S}$ & $\begin{array}{l}\text { Effective thermal Stanton } \\
\text { number }\end{array}$ & $5.9 \times 10^{-4}$ \\
\hline$c_{\rho 1}$ & $\frac{L / c}{C_{d}^{1 / 2} \Gamma_{T S}} \frac{\beta_{T}}{\beta_{S} S_{a}}$ & $2.0 \times 10^{2}$ \\
\hline$c_{\rho 2}$ & $-\lambda_{1} \beta_{T} / \beta_{S}$ & $2.8 \times 10^{-3}$ \\
\hline$c_{\tau}$ & $c_{\rho 2} / c_{\rho 1}$ & $1.4 \times 10^{-5}$ \\
\hline$C_{\varepsilon}$ & Slope correction parameter & 0.6 \\
\hline
\end{tabular}

underestimate the melt-rate maximum by up to $2 \%$ for the test cases considered in section 3, which, however, can increase up to $10 \%$ when the basal slope becomes nearvertical.

Next, we consider an ice-shelf geometry with constant basal slope $\alpha$. Hence, the ice-shelf basal depth $z_{b}$ is related to the coordinate $X$ along the ice-shelf base as follows:

$$
z_{b}=z_{\mathrm{gl}}+X \sin \alpha
$$

where the grounding-line depth $z_{\mathrm{gl}}$ corresponds to $X=0$. Moreover, we also take uniform ambient ocean properties $T_{a}$ and $S_{a}$ (this assumption and its consequences will be discussed further in section $3 b$ ). In particular, we can now define the characteristic freezing point at the grounding line as follows:

$$
T_{f, 0}=\lambda_{1} S_{a}+\lambda_{2}+\lambda_{3} z_{\mathrm{gl}},
$$

which is also constant for a fixed geometry. Note that we have:

$$
T_{f}=T_{f, 0}+\lambda_{1} \Delta S+\lambda_{3} X \sin \alpha,
$$

where $\Delta S=S-S_{a}$ is the salinity contrast between plume and ambient ocean.

Taking into account these assumptions, the plume equations can now be written in a more compact way, expressed only in terms of $D, U, \Delta \rho, \Delta T=T-T_{f}, \Delta S$, and independent variable $X$. Formally, only four equations would be required to close the system, but keeping an equation for $\Delta S$ will simplify the analysis later on. The resulting system has the following closed form:

$$
\begin{aligned}
\frac{d D U}{d X}= & \left(E_{0} \sin \alpha\right) U+\left(\frac{C_{d}^{1 / 2} \Gamma_{T S}}{L / c}\right) U \Delta T \\
\frac{d D U^{2}}{d X}= & \left(\frac{g \sin \alpha}{\rho_{0}}\right) D \Delta \rho-C_{d} U^{2} \\
\frac{d D U \Delta \rho}{d X}= & \rho_{0}\left(\frac{C_{d}^{1 / 2} \Gamma_{T S}}{L / c}\right) U \Delta T\left\{\beta_{S} S_{a}-\beta_{T}\left[T_{a}-T_{f, 0}\right.\right. \\
& \left.\left.+\frac{L}{c}-\lambda_{1} \Delta S-\left(\lambda_{3} \sin \alpha\right) X\right]\right\} \\
\frac{d D U \Delta T}{d X}= & \left(E_{0} \sin \alpha\right) U\left[T_{a}-T_{f, 0}-\left(\lambda_{3} \sin \alpha\right) X\right] \\
& +\left(\frac{C_{d}^{1 / 2} \Gamma_{T S}}{L / c}\right) U \Delta T\left(\lambda_{1} S_{a}+\lambda_{1} \Delta S-\frac{L}{c}\right) \\
& -\left(\lambda_{3} \sin \alpha\right) D U, \quad \text { and } \\
\frac{d D U \Delta S}{d X}= & -S_{a}\left(\frac{C_{d}^{1 / 2} \Gamma_{T S}}{L / c}\right) U \Delta T .
\end{aligned}
$$

Note that these equations only have constant coefficients, as well as some terms that are explicit in $X$ through the depth dependence of the freezing point. Equations (9) can now be nondimensionalized using the following scaling:

$$
\begin{aligned}
& x=\frac{z_{b}-z_{\mathrm{gl}}}{l_{0}}=\left(\frac{\sin \alpha}{l_{0}}\right) X, \\
& d=\frac{D}{E_{0} l_{0}}, \quad u=\frac{U}{U_{0}}, \quad r=\frac{\Delta \rho}{\beta_{S} S_{a} \rho_{0}}, \\
& t=\frac{\Delta T}{\tau}, \quad s=\frac{\Delta S}{S_{a}},
\end{aligned}
$$

where $l_{0}, U_{0}$, and $\tau$ are the characteristic length, velocity, and temperature scales, respectively. These characteristic scales are defined as follows. The temperature difference between ambient ocean $T_{a}$ and the freezing point at the grounding line $T_{f, 0}$ is considered to be the main external parameter that drives the initial melting at the grounding line and, hence, the initial buoyancy flux. Therefore, we take 


$$
\tau=T_{a}-T_{f, 0} .
$$

Neglecting the effects of stratification, Coriolis forces, and initial freshwater fluxes at the grounding line, the main length scale governing the plume dynamics is associated with $T_{a}-T_{f, 0}$ (cf. Lane-Serff 1995; Jenkins 2011; Lazeroms et al. 2018):

$$
l_{0}=\frac{\tau}{\lambda_{3}}
$$

This length scale denotes the vertical distance from the grounding line over which the thermal driving of the plume decreases from the initial value $\tau$ to zero due to the pressure dependence of the freezing point, assuming uniform ambient water. In other words, it indicates the point where the ambient water would start freezing, which may be above or below sea level, depending on ambient conditions (Lane-Serff 1995). Finally, since the dynamics of the plume are determined by melt-induced buoyancy and not by an external flux, the velocity scale is fixed by taking the densimetric Froude number equal to unity:

$$
\mathrm{Fr}=\frac{U_{0}}{\sqrt{\frac{\widetilde{\Delta \rho}}{\rho_{0}} g l_{0}}}=\frac{U_{0}}{\sqrt{\beta_{S} S_{a} g l_{0}}}=1,
$$

where $\widetilde{\Delta \rho}=\beta_{S} S_{a} \rho_{0}$ is the characteristic density scale defined in (10). In addition to these physical scales, we define two dimensionless parameters that determine the entrainment rate and the basal melt rate, respectively:

$$
\eta=E_{0} \sin \alpha, \quad \mu=\frac{C_{d}^{1 / 2} \Gamma_{T S}}{L / c} \tau,
$$

noting that the factor $C_{d}^{1 / 2} \Gamma_{T S} /(L / c)$ is equivalent to the factor $M_{0}$ in Jenkins (2011). Using (10)-(14), the plume equations in (9) can be written in the following way:

$$
\begin{aligned}
\eta \frac{d}{d x}(d u) & =\eta u+\mu u t \\
\eta \frac{d}{d x}\left(d u^{2}\right) & =\eta d r-C_{d} u^{2} \\
\eta \frac{d}{d x}(d u r) & =\mu u t\left[1-c_{\rho 1} C_{d}^{1 / 2} \Gamma_{T S}+\mu c_{\rho 1}(x-1)-c_{\rho 2} s\right]
\end{aligned}
$$

$$
\eta \frac{d}{d x}(d u t)=\eta(1-x-d) u-\left(C_{d}^{1 / 2} \Gamma_{T S}+c_{\tau}+c_{\tau} s\right) u t, \quad \text { and }
$$

$\eta \frac{d}{d x}(d u s)=-\mu u t$ where we introduced a set of new parameters $c_{\rho 1}, c_{\rho 2}$, and $c_{\tau}$, which are defined in Table 1 along with their typical values. The dimensionless system in (15) will now be simplified further using the observation that the parameters $\eta$ and $\mu$ are small. This will lead to an equation that can be solved analytically, yielding a closed expression for the dimensionless basal melt rate.

\section{c. Asymptotic analysis in terms of $\eta$ and $\mu$}

A central notion in perturbation theory is the asymptotic expansion of order $N$ (or Poincaré expansion; see, e.g., Nayfeh 1973; Eckhaus 1979; Holmes 1995; Mattheij et al. 2005) of a function $f(x ; \varepsilon)$ in terms of a small problem parameter $\varepsilon \ll 1$ :

$f(x ; \varepsilon) \approx \sum_{n=0}^{N} a_{n}(\varepsilon) f_{n}(x), \quad$ with $\lim _{\varepsilon \rightarrow 0} \frac{a_{n+1}(\varepsilon)}{a_{n}(\varepsilon)}=0$,

in which each consecutive term $a_{n}(\varepsilon)$ decreases strictly faster as $\varepsilon$ goes to zero [e.g., $a_{n}(\varepsilon)=\varepsilon^{n}$ ]. Ideally, this allows the asymptotic expansion to converge to the function $f(x ; \varepsilon)$ for $\varepsilon \rightarrow 0$. Such an expansion can be used to systematically approximate problems that are too complicated to solve analytically. As we shall see below, it is often convenient to scale the equations by the leadingorder asymptote $a_{0}(\varepsilon)$ so that the first term in the expansion is $O(1)$ as $\varepsilon \rightarrow 0$. This allows us to expand all dependent variables individually.

System (15) contains two parameters that are potentially small and that are dependent on external properties, namely $\eta$ (depending on the basal slope) and $\mu$ (depending on the temperature difference $\tau$ ). Since we disregard changes in the ambient salinity $S_{a}$, the remaining parameters in (15) are model constants with values given in Table 1. Before we proceed, it is worthwhile investigating if $\eta$ and $\mu$ are indeed small for typical ice-shelf conditions and if they are of the same order of magnitude. With ambient salinity $S_{a}=34.65 \mathrm{psu}$ and a typical grounding-line depth $z_{\mathrm{gl}} \geq-1500 \mathrm{~m}$, a lower bound for the freezing point $T_{f, 0}$ is $-3^{\circ} \mathrm{C}$. With typical ambient temperatures $T_{a}$ between $-2^{\circ}$ and $+2^{\circ} \mathrm{C}$, this yields upper bounds $\tau<5^{\circ} \mathrm{C}$ and $\mu<3.5 \times 10^{-5}$, which is indeed small. A typical estimate of the basal slope $\sin (\alpha)$ is more difficult to find, as the slope can vary considerably along the ice shelf. As $\sin (\alpha)$ cannot exceed 1, a strict upper bound is $\eta<E_{0}=3.6 \times 10^{-2}$. Hence, both $\eta$ and $\mu$ are indeed small parameters.

It appears that $\mu$ is potentially some orders of magnitude smaller than $\eta$, although one should keep in mind that small values of $\sin (\alpha)$ are very common for most ice shelves, even close to the grounding line. For example, we can consider the overall slope of a flowline of Filchner-Ronne Ice Shelf (FRIS), taking $z_{\mathrm{gl}} \approx-1500 \mathrm{~m}$ and a horizontal 
distance of $700 \mathrm{~km}$, yielding $\sin (\alpha) \approx 2.1 \times 10^{-3}$ and $\eta \approx 7.7 \times 10^{-5}$ [see, e.g., the data of Bombosch and Jenkins (1995) used in Lazeroms et al. (2018) and the test cases in Fig. 8]. Taking $T_{a} \approx-2^{\circ} \mathrm{C}$ as a typical ambient temperature, we obtain $\tau \approx 1^{\circ} \mathrm{C}$ and $\mu \approx 7 \times 10^{-6}$. Similarly, we could estimate the slope of Pine Island Glacier (PIG, also shown in Fig. 8) as $\sin (\alpha) \approx 500 \mathrm{~m} / 60 \mathrm{~km} \approx$ $8.3 \times 10^{-3}$, giving $\eta \approx 3 \times 10^{-4}$, while $\mu$ might approach the upper bound $3.5 \times 10^{-5}$ for warm cavities mentioned above. In these cases, $\eta$ is indeed one order of magnitude larger than $\mu$, also considering that the slope near the grounding line is typically higher than the overall slope. This would justify the scaling assumption that terms of $O(\mu)$ in (15) could be neglected with respect to terms of $O(\eta)$. Physically, this means neglecting the direct effect of the melt rate on the volume flux in (15a) with respect to the entrainment rate. Note that this condition might be violated locally for relatively warm ice shelf cavities with small slopes.

The next step is to determine how the dependent variables $(d, u, r, t, s)$ scale with $\eta$ and $\mu$, that is, determine the scale function $a_{0}(\eta, \mu)$ in analogy to (16) for each variable. This can be done by inspection of (15), requiring that every equation retains a meaningful balance of terms in the limits $\eta \rightarrow 0$ and $\mu \rightarrow 0$. A crucial observation here is that $x=O(1)$ by construction, since otherwise $l_{0}$ would not be the governing length scale. This allows us to estimate the magnitudes of the terms in (15a) as $\eta d u, \eta u$, and $\mu u t$. Assuming that $d, u, r, t, s \leq O(1)$ and $O(\mu)<O(\eta)$ as explained earlier, we see that the final term, the melt rate, is indeed neglected at leading order. Similar arguments can be applied to the other terms in (15), where the constant parameters such as $C_{d}$ are considered $O(1)$ because they are independent of $\eta$ and $\mu$. From top to bottom, we then find the following leading-order balance of terms:

$$
\begin{gathered}
\eta d u \sim \eta u, \\
\eta d r \sim u^{2}, \\
\eta d u r \sim \mu u t, \\
\eta u \sim u t, \\
\eta d u s \sim \mu u t,
\end{gathered}
$$

which yields

$$
\begin{aligned}
d & =O(1), \quad u=O\left[(\eta \mu)^{1 / 2}\right], \quad r=O(\mu), \\
t & =O(\eta), \quad s=O(\mu) .
\end{aligned}
$$

Physically, this means that both the density and salinity differences scale with the melt rate $\mu$, while the temperature difference depends on the slope $\eta$. As it turns out, all terms in (15) depending explicitly on the salinity difference $s$ will be of higher order in $\mu$ and neglected in the following approximation. Consequently, $s$ and its corresponding primitive equation (15e) can be disregarded.

We can now extract the scales in (18) and expand to leading order in $\mu$. Furthermore, we incorporate several algebraic factors that are essentially $O(1)$ functions of $\eta$, which make the resulting equations more compact. This amounts to the following scaling:

$d=\mathscr{D}+O(\mu)$,

$u=(\eta \mu)^{1 / 2}\left[\frac{1-c_{\rho 1} C_{d}^{1 / 2} \Gamma_{T S}}{\left(C_{d}+\eta\right)\left(C_{d}^{1 / 2} \Gamma_{T S}+c_{\tau}+\eta\right)}\right]^{1 / 2}[\mathscr{C}+O(\mu)]$,

$r=\mu\left(\frac{1-c_{\rho 1} C_{d}^{1 / 2} \Gamma_{T S}}{C_{d}^{1 / 2} \Gamma_{T S}+c_{\tau}+\eta}\right)[\mathscr{B}+O(\mu)], \quad$ and

$t=\eta\left(\frac{1}{C_{d}^{1 / 2} \Gamma_{T S}+c_{\tau}+\eta}\right)[\mathscr{T}+O(\mu)]$,

By substituting these expression in (15) and dividing by common factors, we then obtain the following system, valid up to $O(\mu)$ :

$$
\begin{aligned}
\frac{d}{d x}(\mathscr{D} \mathscr{C}) & =\mathscr{W}, \\
\delta(\eta) \mathscr{D} \mathscr{C} \frac{d \mathscr{C}}{d x} & =\mathscr{D} \mathscr{B}-\mathscr{C}^{2}, \\
\frac{d}{d x}(\mathscr{D} \mathscr{C} \mathscr{R}) & =\mathscr{W} \mathscr{T}, \quad \text { and } \\
\varepsilon(\eta) \mathscr{D} \frac{d \mathscr{T}}{d x} & =1-x-\mathscr{D}-\mathscr{T} .
\end{aligned}
$$

with

$$
\delta(\eta)=\frac{\eta}{C_{d}+\eta}, \quad \varepsilon(\eta)=\frac{\eta}{C_{d}^{1 / 2} \Gamma_{T S}+c_{\tau}+\eta}
$$

where we have also used the mass equation (20a) to expand the derivatives in the momentum and heat equations (20b) and (20d). Note that this system is indeed uncoupled from the salinity equation, so that it can be disregarded in the remainder of this study. This also clarifies the reason for initially keeping the salinity equation, as mentioned in section $2 \mathrm{a}$, because it simplifies the scaling analysis done so far. Furthermore, we see that the advection terms in the mass and buoyancy equations (20a) and (20c) are of leading order, while those in the 
momentum and heat equations are $O(\eta)$, which will have important consequences further on.

We have now scaled the plume equations to a compact form containing only $O(1)$ terms except for the velocity and temperature derivatives, which have new factors $\delta(\eta)$ and $\varepsilon(\eta)$. What remains is the question of whether $\delta(\eta)$ and $\varepsilon(\eta)$ are still small parameters and if we can use an asymptotic expansion in either $\delta$ or $\varepsilon$ to further simplify the analysis. Figure 2 shows these parameters as a function of $\eta$ within a range of values deemed typical for ice shelves (the previously found values for FRIS and PIG are indicated in the figure, as well as a typical value for Ross ice shelf). It appears that $\varepsilon$ is always larger than $\delta$ and larger than 0.1 over a significant range of values. Still, $\varepsilon$ could be considered as a small parameter except for the larger values of $\eta$. Furthermore, although formally both $\varepsilon$ and $\delta$ scale with $\eta$ and their ratio is approximately constant, it appears that $\delta$ is one order of magnitude smaller than $\varepsilon$ over a wide range of values except for very small $\eta \lesssim 10^{-5}$. This would justify a second assumption $\delta<O(\varepsilon)$, making the momentum advection term in (20b) one order smaller than the heat advection term in (20d).

Equations (20) describe the behavior of the dimensionless quantities $(\mathscr{D}, \mathscr{C}, \mathscr{R}, \mathscr{T})$ as a function of the dimensionless coordinate $x$. Since the melt rate $\dot{m}$ is essentially the product $U \Delta T$ [see (5a)], the scaled product $\mathscr{C}=\mathscr{C} \mathscr{C} \mathscr{T}$ describes the dimensionless melt rate. To leading order in $\varepsilon$ and $\mu,(20)$ should be interpreted as follows: (20a) is the mass balance determined entirely by entrainment with meltwater input neglected; (20b) is a balance of buoyancy and drag forces, neglecting inertial accelerations up to $O(\delta) ;(20 \mathrm{c})$ is the buoyancy flux determined entirely by the melt rate, neglecting higher-order changes in plume temperature and plume salinity; and (20d) determines the plume temperature contrast from the depth-dependent freezing point, entrainment, and the ice-ocean interface heat flux, neglecting higher-order changes in plume salinity and melt rate, while downstream changes in temperature are $O(\varepsilon)$. In the next subsection, we show how system (20) can be solved with an asymptotic expansion in $\varepsilon$. Note that the error in this approach with respect to the full plume model depends on the value of $\varepsilon$ (i.e., the basal slope). The extent of the error in realistic test cases will become clear in section 3 .

\section{d. Analytical expression for the melt rate}

The structure of the solution of (20) is most easily understood by writing it as a single equation. It turns out that the volume flux, defined as

$$
\varphi=\mathscr{D} \mathscr{C},
$$



FIG. 2. Logarithmic plots of the parameters $\delta$ and $\varepsilon$ defined in (21) and their ratio $\delta / \varepsilon$ as a function of the entrainment parameter $\eta$. The vertical dashed lines indicates the typical value for Ross $\left(\eta \approx 2.6 \times 10^{-5}\right)$, FRIS $\left(\eta \approx 7.7 \times 10^{-5}\right)$, and PIG $\left(\eta \approx 3 \times 10^{-4}\right)$.

is the most convenient variable to use, for example, because (20a) then becomes $d \varphi / d x=\mathscr{C}$, directly expressing the dimensionless velocity in terms of $\varphi$. In appendix A, we show how system (20) can be reduced to the following equation with $\delta=\varepsilon^{2}$ :

$$
(1-\varepsilon)\left(\varphi^{\prime}\right)^{3}-\varphi\left[1-x-\varepsilon(3+\varepsilon) \varphi^{\prime} \varphi^{\prime \prime}\right]+O\left(\varepsilon^{3}\right)=0,
$$

where the primes indicate derivatives with respect to $x$. Moreover, we have initial conditions $\varphi(0)=\varphi^{\prime}(0)=0$. The first condition simply corresponds to zero initial mass flux, that is, $D U=0$ at the grounding line, while the condition $\varphi^{\prime}(0)=0$ corresponds to $U(0)=0$, which is implied by (20b). This is the most compact formulation of the problem. Correspondingly, we find an expression in terms of $\varphi$ for the dimensionless melt rate:

$$
\mathscr{L}=3\left(\varphi^{\prime}\right)^{2} \varphi^{\prime \prime}+O\left(\varepsilon^{2}\right) .
$$

Note that the problem is now reduced to a second-order differential equation for $\varphi$, requiring only two boundary conditions. Hence, the boundary values for the plume temperature and plume salinity are automatically imposed by the solution of (23) and cannot be chosen independently. Physically, the initial meltwater at the grounding line undergoes a rapid adjustment as it mixes with the ambient water, a process also described by Jenkins (2011). By reducing the order of the system and the number of boundary conditions, we neglect this rapid adjustment and immediately start from the mixed conditions.

An analytical expression for the melt rate can be obtained from (24) after finding the solution of (23). As explained in detail in appendix A, an analytical solution will be constructed using an asymptotic expansion of 
$\varphi$ in terms of $\varepsilon$. Restricting our attention to the leadingorder behavior, the positive real-valued solution for $0 \leq x \leq 1$ is found to be

$$
\varphi_{0}(x)=\frac{1}{2 \sqrt{2}}\left[1-(1-x)^{4 / 3}\right]^{3 / 2} .
$$

Note that the function $\varphi_{0}$ has an important physical meaning: it is the scaled volume flux of ambient water in the plume that determines the strength of the overturning circulation inside the cavity, making it a key quantity for parameterizing the effects of ice-shelf melting in ocean models. Furthermore, as explained in appendix A, this leading-order solution is equivalent to the assumption that the volume fraction of meltwater in the plume $\left(\varphi_{0}^{3}\right)^{\prime} / \varphi_{0}$ falls linearly as $1-x$, which can be seen by taking $\varepsilon \rightarrow 0$ in (23). From (25) and (24), we can directly obtain an expression for the melt rate:

$$
\begin{aligned}
\mathscr{C l}_{0}(x) & =3\left[\varphi_{0}^{\prime}(x)\right]^{2} \varphi_{0}^{\prime \prime}(x) \\
& =\frac{1}{2 \sqrt{2}}\left[3(1-x)^{4 / 3}-1\right]\left[1-(1-x)^{4 / 3}\right]^{1 / 2} .
\end{aligned}
$$

We now have the main result of the derivation, namely an explicit analytical expression for the dimensionless melt rate. Physically, it describes the basal melt rates along the plume path after applying an appropriate scaling in terms of the basal slope $\eta$ and the thermal driving of the plume $\mu$. In this respect, it should be noted that (26) takes over the role of the dimensionless melt-rate curve applied by Lazeroms et al. (2018), which was described by a polynomial fit and found from a purely empirical study (Jenkins 2014). The expressions for the other plume variables $\mathscr{D}_{0}, \mathscr{C}_{0}, \mathscr{B}_{0}$, and $\mathscr{T}_{0}$, as well as interesting points about their physical interpretation, are given in appendix A.

Figure $3 \mathrm{a}$ shows a plot of the analytical expression (26) as a function of the dimensionless coordinate $x$. The melt-rate curve indeed shows the desired behavior: the melt rate is zero at the grounding line $(x=0)$ and increases to a positive peak at $x \approx 0.2$ before transitioning to negative melt (refreezing) around $x \approx 0.6$. This transition point agrees with the empirical value found by Lane-Serff (1995). The same qualitative behavior is seen in the empirical melt-rate curve applied in Lazeroms et al. (2018), also shown in Fig. 3a. However, since (26) represents only a leading-order asymptotic approximation of (23) and (24), it is independent of $\varepsilon$. Therefore, the errors with respect to the (numerical) solution of these original equations will depend on $\varepsilon$, and it is important to check how these errors are distributed over the entire domain of interest. Numerical solutions for (a)

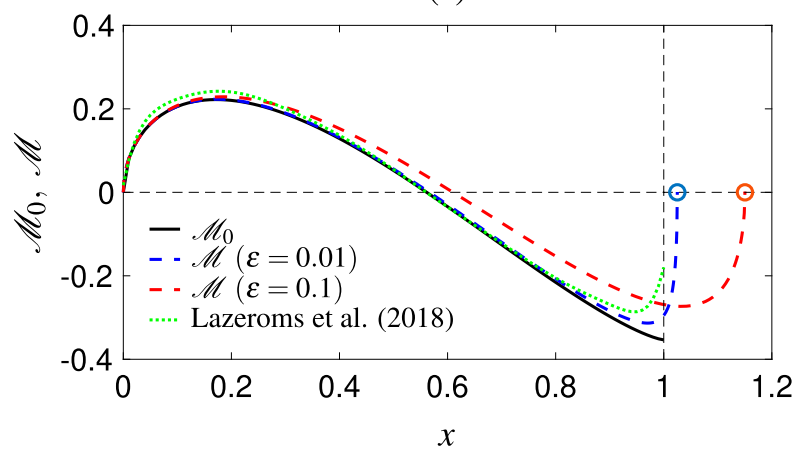

(b)

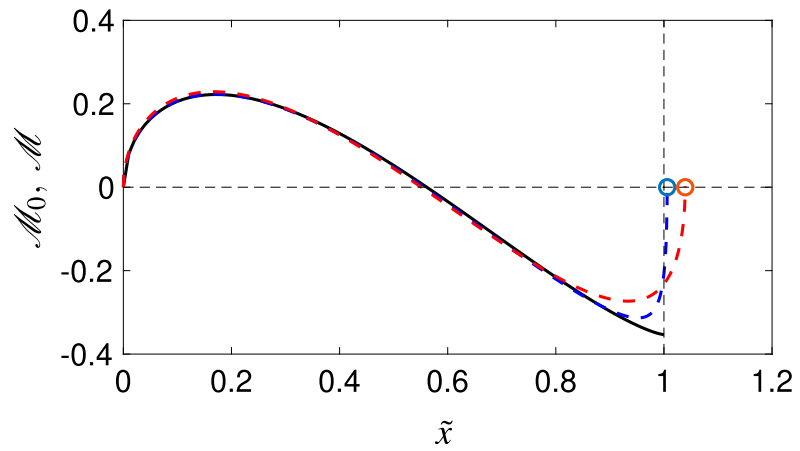

FIG. 3. Comparison of the analytical solution for $\mathscr{C l}_{0}$ calculated from (26) (solid line) with numerical solutions $\mathscr{C} 6$ calculated from (23) and (24) (dashed lines). Also shown is the polynomial fit from Lazeroms et al. (2018) scaled with a constant factor 0.124 (dotted line). Each panel shows two numerical solutions for $\varepsilon=0.01$ and $\varepsilon=0.1$, respectively. (a) Solutions as functions of the original dimensionless coordinate $x$. (b) Solutions as functions of the new coordinate $\tilde{x}$ containing the slope correction of (27). Note that the analytical solution and the polynomial fit are valid on the domain $[0,1]$, while the numerical solutions are valid until the plume endpoints indicated by circles.

$\varepsilon=0.01$ and $\varepsilon=0.1$ are shown in Fig. 3a and directly compared with the analytical expression. Clearly, the analytical expression approximates the numerical curves well close to the grounding line $(x=0)$, but the discrepancies become larger further away from the grounding line and increase with $\varepsilon$. In fact, the analytical expression (26) is only valid for $0 \leq x \leq 1$, while the numerical solutions continue until the points $x \approx 1.025$ and $x \approx 1.15$, respectively. Hence, the "endpoint" of the plume moves further away from $x=1$ as $\varepsilon$ increases. Conversely, the endpoint moves toward $x=1$ for $\varepsilon \rightarrow 0$, in which case the numerical solution converges to expression (26) for $0 \leq x \leq 1$.

So far, we have only considered the zeroth-order terms in the asymptotic expansion. One might expect to improve the approximation by adding higher-order terms in our asymptotic expansion. However, this turns 
out not to be possible for technical reasons explained in appendix B. On the other hand, some of the aforementioned discrepancies might be overcome by adding an additional slope-dependent correction, similar to the ad hoc scaling used by Jenkins (2014) and Lazeroms et al. (2018). Intuitively, such an additional $\varepsilon$-dependent correction should provide the final scaling step that constrains the values of the along-slope coordinate between 0 and 1 , where the latter corresponds to the aforementioned $\varepsilon$-dependent endpoint of the plume. The theoretical discussion in appendix B suggests a correction of the following form:

$$
\tilde{x}=\frac{x}{1+C_{\varepsilon} \varepsilon^{3 / 4}}
$$

where $C_{\varepsilon}=0.6$ turns out to give the best match between the analytical expression and the numerical solution of (23) for $\varepsilon<0.3$. Figure $3 \mathrm{~b}$ shows how the results from Fig. 3a change when the additional correction is applied. The three curves now agree well in most of the domain, except for a small region near $\tilde{x}=1$. Note, however, that this region may not be important in practice for the following reasons. The point $x=1$ (i.e., the original coordinate) corresponds exactly to a depth at which $z_{b}-z_{\mathrm{gl}}=l_{0}$, that is, where $T_{a}$ is equal to the local ambient freezing point. It appears unphysical to have ambient waters colder than the local freezing point, except for, for example, locally supercooled plumes with possible nucleation of frazil ice crystal, which is not captured by the plume model equations in (1). Therefore, the point $x=1$ can only be reached at the surface $z_{b}=0$ when $T_{a}$ is equal to the ambient freezing temperature at atmospheric pressure. For higher $T_{a}$, the point $x=1$ occurs above the sea surface and is clearly nonphysical. Moreover, the point $x=1$ corresponds to a corrected value $\tilde{x}<1$ for positive slopes $\varepsilon>0$, making it more unlikely for the model to approach the point $\tilde{x}=1$, and increasingly so for steeper slopes. The results in section 3 indeed show that the true endpoint of the plume always has a value $\tilde{x} \in(0,1)$ and the discrepancies remain small or outranked by other effects such as varying slopes or stratification. Most importantly, the correction in (27) ensures that the transition point between melting and refreezing is almost perfectly predicted for $\varepsilon<0.3$.

To summarize, we have systematically derived an analytical expression (26) for the dimensionless basal melt rate as a function of the along-slope coordinate under an ice-shelf with constant slope and constant ambient properties. We can trace back the derivation to the dimensional quantities and arrive at the final form of the proposed basal melt parameterization, including dimensional factors:

$$
\begin{aligned}
\dot{m}= & \sqrt{\frac{\beta_{S} S_{a} g}{\lambda_{3}(L / c)^{3}}}\left(\frac{1-c_{\rho 1} C_{d}^{1 / 2} \Gamma_{T S}}{C_{d}+E_{0} \sin \alpha}\right)^{1 / 2} \\
& \times\left(\frac{C_{d}^{1 / 2} \Gamma_{T S} E_{0} \sin \alpha}{C_{d}^{1 / 2} \Gamma_{T S}+c_{\tau}+E_{0} \sin \alpha}\right)^{3 / 2}\left(T_{a}-T_{f, 0}\right)^{2} \mathscr{L}_{0}(\tilde{x}),
\end{aligned}
$$

where $\mathscr{C}_{0}$ is the analytical function in (26) with input $\tilde{x}$ given by

$\tilde{x}=\lambda_{3} \frac{z_{b}-z_{\mathrm{gl}}}{T_{a}-T_{f, 0}}\left[1+C_{\varepsilon}\left(\frac{E_{0} \sin \alpha}{C_{d}^{1 / 2} \Gamma_{T S}+c_{\tau}+E_{0} \sin \alpha}\right)^{3 / 4}\right]^{-1}$.

Similarly, we can express the dimensional volume flux $\Phi=D U$ of the cavity circulation in terms of the function $\varphi_{0}$ in (25):

$$
\begin{aligned}
\Phi= & E_{0} \sqrt{\frac{\beta_{S} S_{a} g}{\lambda_{3}^{3}(L / c)}}\left(\frac{1-c_{\rho 1} C_{d}^{1 / 2} \Gamma_{T S}}{C_{d}+E_{0} \sin \alpha}\right)^{1 / 2} \\
& \times\left(\frac{C_{d}^{1 / 2} \Gamma_{T S} E_{0} \sin \alpha}{C_{d}^{1 / 2} \Gamma_{T S}+c_{\tau}+E_{0} \sin \alpha}\right)^{1 / 2}\left(T_{a}-T_{f, 0}\right)^{2} \varphi_{0}(\tilde{x}) .
\end{aligned}
$$

These expressions should be compared with the basalmelt parameterization found empirically by Jenkins (2014) and applied to the Antarctic ice shelves by Lazeroms et al. (2018). In both formulations, the basal melt is calculated by multiplying a melt-rate scale by a dimensionless function $\mathscr{1}_{0}$ of a dimensionless coordinate $\tilde{x}$. In fact, the melt-rate scale in (28a) is almost equal to the empirical scale shown in Lazeroms et al. (2018), being a product of geometric factors involving $\sin (\alpha)$ and a quadratic dependence on $T_{a}-T_{f, 0}$. A notable difference is the addition of some new constants that only have a very small effect on the final value of $\dot{m}$ (e.g., the constant $c_{\tau}$ with respect to $C_{d}^{1 / 2} \Gamma_{T S}$ ). Moreover, the constant prefactor in (28a) is now explicitly given in terms of other model constants, whereas Lazeroms et al. (2018) simply used a parameter $M_{0}=10 \mathrm{~m} \mathrm{yr}^{-1}{ }^{\circ} \mathrm{C}^{-2}$. Apart from this, (28a) contains the same dependence on $\sin (\alpha)$ with the same exponents as in the empirical model. Similarly, the dimensionless coordinate in (28b) has the same basic form as in the empirical model: the depth difference $z_{b}-z_{\mathrm{gl}}$ scaled by the temperature difference $T_{a}-T_{f, 0}$ and multiplied by a slope-dependent factor. This factor differs slightly from the one in Lazeroms et al. (2018), but in both cases it is an ad hoc correction with the purpose of constraining the coordinate values 
between 0 and 1 for different values of the slope. Finally, the function $\mathscr{C}_{0}$ given by (26) now plays the same role as the dimensionless polynomial fit in Lazeroms et al. (2018). Note, however, that the analytical expression (26) and the polynomial fit differ by a factor of 0.124 (as shown in Fig. 3a) due to the aforementioned difference in the constant dimensional prefactor. In the next section, we show how the basal-melt parameterization given by (28) performs for various ice-shelf geometries and ambient ocean properties.

\section{Numerical results}

So far, the analytical expression for the melt rate given by (28a) has been directly compared with (23) from which it is derived. Since this equation is itself an approximation of the plume model, it is important to check how it compares to the full model. In the following, we shall evaluate the plume model as presented in section $2 \mathrm{a}$ and the parameterization given by (28) for various test cases. Both models are evaluated using the constant parameter values given in Table 1 . We distinguish between effects of the geometry and the ambient ocean profiles, before applying the model to more realistic cases.

\section{a. Slope dependence}

The main assumptions of the derivation as formulated in section $2 \mathrm{~b}$ are a constant slope angle $\alpha$ (i.e., a linear ice-shelf draft) and uniform ambient properties $T_{a}$ and $S_{a}$. First, we investigate how well the analytical expression (28) agrees with the full plume model [including the three-equation formulation in (4)] for such simplified cases. In the following, both models are evaluated for simple geometries defined by the grounding-line depth $z_{\mathrm{gl}}$ and a slope angle $\alpha$ and extending up to sea level $(z=0)$. The reference geometry has $z_{\mathrm{gl}}=-1000 \mathrm{~m}$ and $\sin (\alpha)=0.002$, so that the plume path extends up to $X=500 \mathrm{~km}$. By order of magnitude, this is comparable to the larger Antarctic ice shelves such as FRIS and Ross ice shelf. Moreover, we consider steep and flat geometries $[\sin (\alpha)=0.003$ and $\sin (\alpha)=0.001$, respectively] and shallow and deep geometries $\left(z_{\mathrm{gl}}=-500 \mathrm{~m}\right.$ and $z_{\mathrm{gl}}=-2000 \mathrm{~m}$, respectively), as well as the special case of a vertical ice wall $\left[\sin (\alpha)=1\right.$ and $\left.z_{\mathrm{gl}}=-500 \mathrm{~m}\right]$. In each case, the plume model is evaluated up to the iceshelf front at $z=0$, though in section $3 b$ we will show examples where the plume path ends before reaching the end of the domain.

Figure 4 a shows the results of both models for all of the aforementioned geometries. Clearly, in each case the parameterization closely follows the behavior of the plume model over the entire domain. All curves, except the vertical ice wall, show the same qualitative behavior outlined in section $2 \mathrm{~d}$ : a region of melting (positive $\dot{m}$ ) closer to the grounding line and a transition to refreezing (negative $\dot{m}$ ) further away from the grounding line. A steeper slope causes an increase in melt near the grounding line, as well as stronger refreezing further away, due to stronger volume flux and entrainment, while the flatter slope has the opposite effect. Likewise, a deeper grounding line causes both increased melting and refreezing due to the lower freezing point and an associated increase of thermal driving at greater depths, with the shallow case showing the opposite. In all cases, relatively small discrepancies between the models appear both at the melting peaks closer to the grounding line and near the end of the domain in the area of refreezing, while the transition point between melting and refreezing is almost perfectly predicted by the parameterization.

The similarities between the first five cases become clear in Fig. 4b, where the plume model results with corresponding $X$ coordinates have been scaled using the same dimensional factors as in (28a) and (28b) and directly compared with the dimensionless curve $\mathscr{C}_{0}(\tilde{x})$. The plume model results collapse onto nearly the same curve, which is again slightly underpredicted by the parameterization at the melting peak $(\tilde{x} \approx 0.2)$. The typical relative error in the melting region for these five cases is around $10 \%$ (disregarding the small regions where the melt rate goes to zero). This particular error can be traced back to the fact that we only use a zeroth-order approximation in $\varepsilon$ and might be improved by higher-order terms. Also note that this error depends on $\varepsilon$ and will be smaller for smaller basal slopes. On the other hand, the errors near the end of the domain $(\tilde{x} \approx 0.9)$ are related to the singularities discussed in appendixes $\mathrm{A}$ and $\mathrm{B}$ and are not easily improved, though they appear relatively small with relative values between $0 \%$ and $10 \%$. Note that the good agreement around the melting-freezing transition point $(\tilde{x} \approx 0.56)$ is partly the result of the correction term (27) that rescales the plume-path coordinate.

Among the results in Fig. 4, the vertical ice wall is a special case because it has an infinitely steep slope with $\sin (\alpha)=1$. For such high values of the slope, the parameter $\varepsilon$ in (21) approaches 1 and is no longer small. Hence, the analysis of the previous section is no longer valid for this case. This issue appears in Fig. 4 through the slightly higher discrepancies between the plume model and the parameterization. The relative error in this cases now reaches values between $20 \%$ and $30 \%$. Nevertheless, both models are still reasonably close, which shows that the parameterization is useful even for high slopes and at the calving fronts of ice shelves and tidewater glaciers.

Since the constant-slope assumption will generally not hold for realistic ice-shelf geometries, the next step is to 
(a)

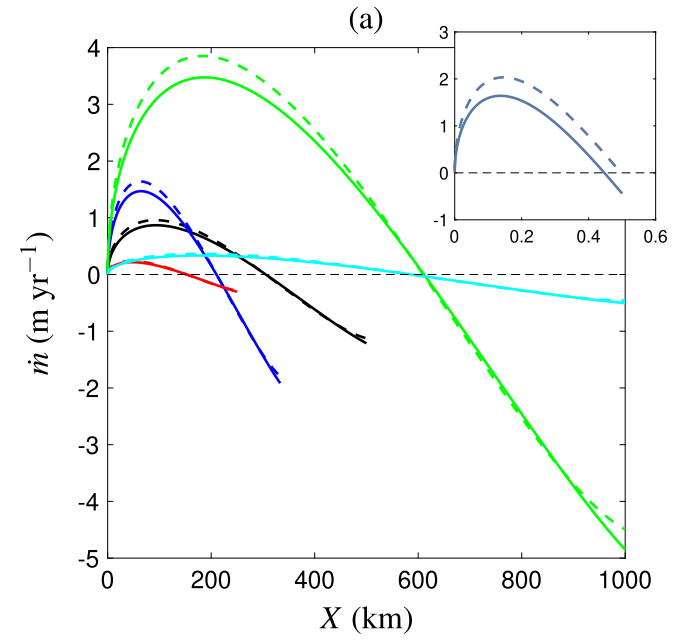

(b)

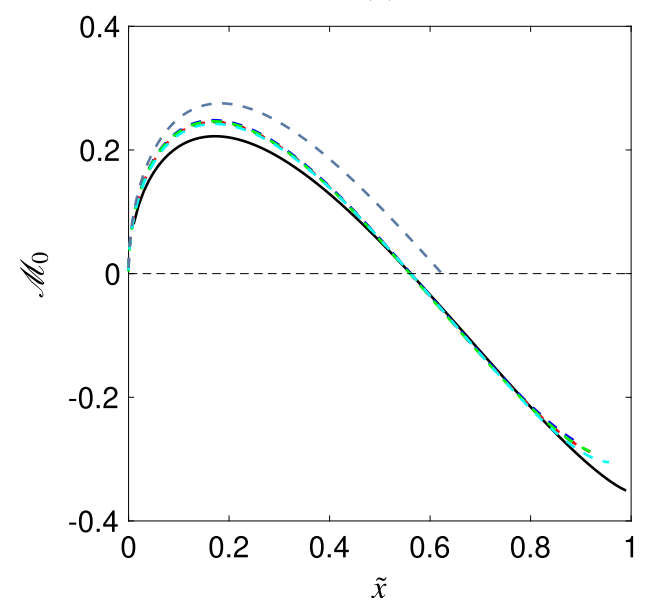

FIG. 4. Comparison of the basal melt rates obtained from (28) (solid lines) and the full plume model (dashed lines) for different idealized ice-shelf geometries with constant slope and uniform ambient properties $\left(S_{a}=34.65 \mathrm{psu}, T_{a}=-1.9^{\circ} \mathrm{C}\right)$. (a) Unscaled results. (b) Scaled plume model results compared with the dimensionless melt-rate curve $\mathscr{C}_{0}(\tilde{x})$. The used geometries are reference geometry (black) with $z_{\mathrm{gl}}=-1000 \mathrm{~m}$ and $\sin (\alpha)=0.002$, steep geometry (blue) with $\sin (\alpha)=0.003$, flat geometry (cyan) with $\sin (\alpha)=0.001$, shallow geometry (red) with $z_{\mathrm{gl}}=-500 \mathrm{~m}$, deep geometry (green) with $z_{\mathrm{gl}}=-2000 \mathrm{~m}$, and [inset in (a)] vertical ice wall (gray) with $z_{\mathrm{gl}}=-500 \mathrm{~m}$ and $\sin (\alpha)=1$. Note that in all cases the curves extend up to the end of the ice-shelf base at sea level.

investigate the performance of the parameterization for varying slopes. Although this goes beyond the formal assumptions behind its derivation, it is possible to evaluate (28) by inserting a varying slope $\alpha(X)$ directly in the equations. Here, we consider four geometries: a convex shelf (increasing $\alpha$ ), a concave shelf (decreasing $\alpha$ ), a shelf with a periodically varying slope, and a shelf consisting of two line segments with different slopes (abrupt change in $\alpha$ ). A comparison of the parameterization and the full plume model for these cases is shown in Fig. 5. In all cases, the parameterization still appears to give results close to the plume model, even though the scaled plume model results can differ considerably from the dimensionless curve $\mathscr{L}_{0}(\tilde{x})$.

In particular, comparing Figs. 5a and 5d, we see that both the height of the melting peak and the error here are higher for the concave case, which starts with a high slope at the grounding line, than for the convex case. Due to the slope-dependent scaling in (28b), the melting peak is also closer to the grounding line in the concave case. Though the melting-freezing transition is almost perfectly predicted in both cases, a larger discrepancy remains in the refreezing region of Fig. 5d. This could be explained by considering that the discrepancies between the dynamically evolving plume model and the parameterization are typically higher in regions where the slope changes, because the plume model adapts more gradually to such changes. Having these slope changes in the refreezing region close to the inherent singularity at $\tilde{x}=1$, as shown in the concave case, will also contribute to these errors.

An interesting example is shown in Fig. $5 \mathrm{~g}$ because the parameterization remains close to the original plume model despite the rapid slope changes over the entire length of the ice shelf. This case clearly shows that high melt rates are obtained locally where the slope is relatively steep. Hence, although the dimensionless curve $\mathscr{C}_{0}(\tilde{x})$ has only one positive melting peak, the unscaled parameterization yields many melting peaks. These peaks are located at the same positions as in the plume model, but the plume model again adapts more gradually to the slope changes. Therefore, the relative error in $\mathscr{A}_{0}$ is highest in regions where the slope flattens, though this has only a minor impact because the melt rates are small here. A similar behavior is shown in Fig. 5j for the abruptly changing slope. As the slope suddenly increases, the parameterization predicts a discontinuous jump in the melt rate whereas the plume model adapts more gradually.

The examples above show that the parameterization formulated in (28) agrees well with the original plume model, not only for simple constant-slope geometries but also for more complicated cases. Despite the presence of errors, which can be explained quite easily from the assumptions in the derivation, the agreement appears good enough to apply the parameterization to more realistic geometries.

\section{b. Thermal driving and stratification}

Next, we investigate the effect of the temperature and the salinity of the ambient ocean water inside the iceshelf cavity. The thermal driving, that is, the temperature difference $T_{a}-T_{f, 0}$ at the grounding line, is perhaps the most important input variable determining the absolute value of the melt rates in (28a). Figure 4 already 
(a)

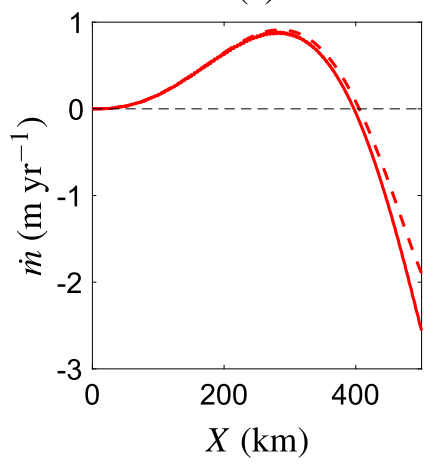

(d)

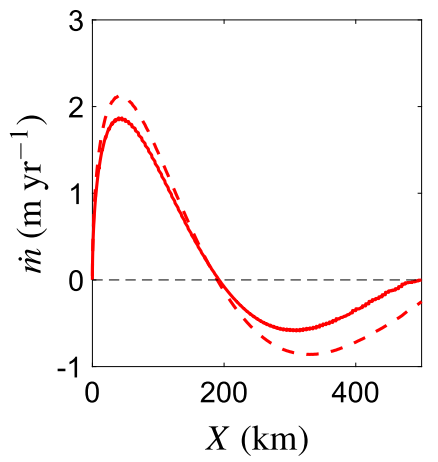

(g)

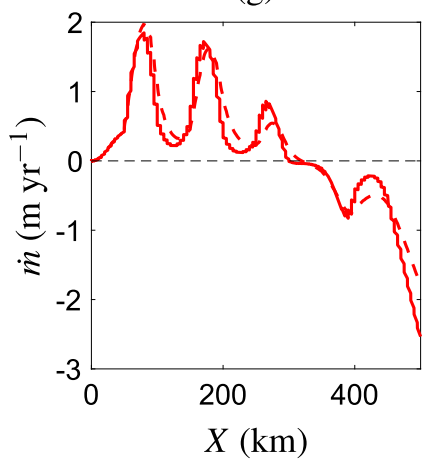

(j)

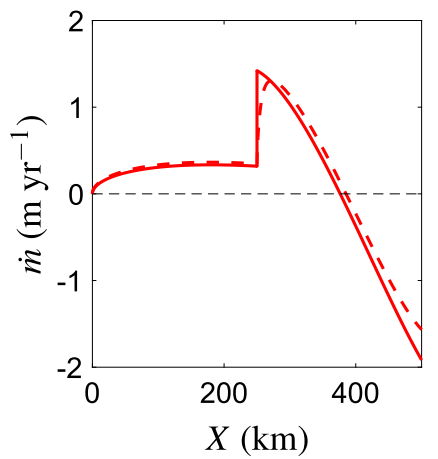

(b)

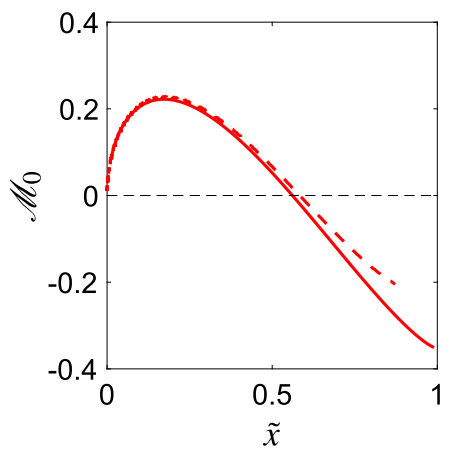

(e)

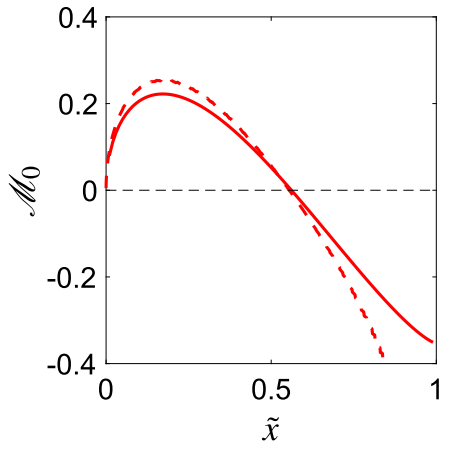

(h)

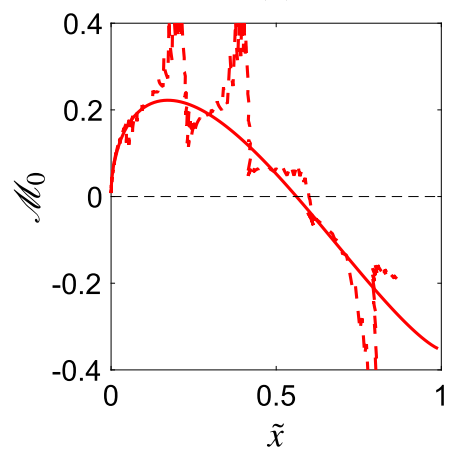

$(\mathrm{k})$

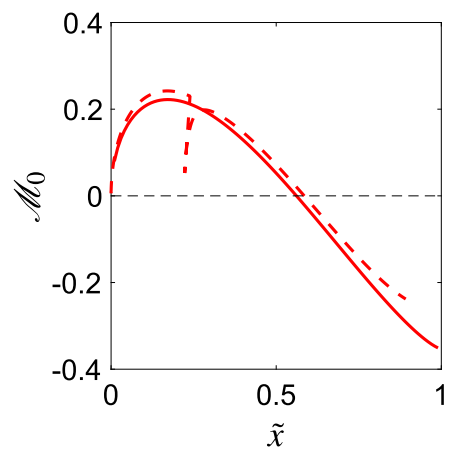

(c)



(f)

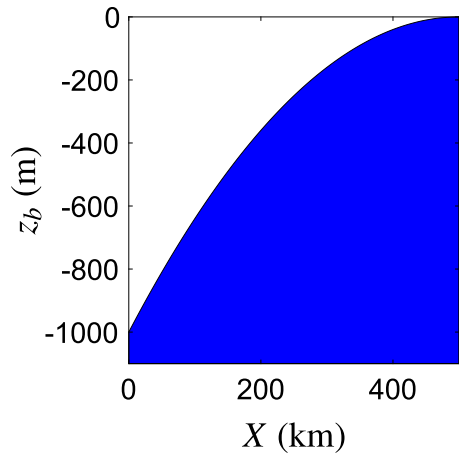

(i)

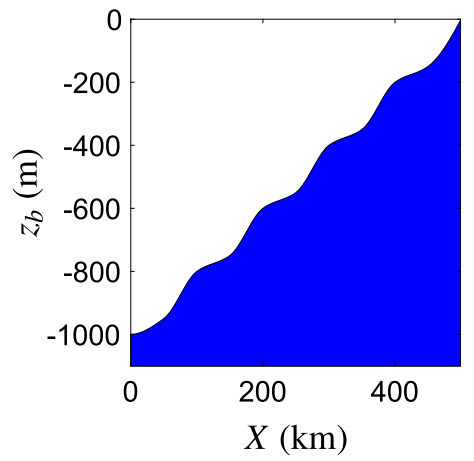

(1)

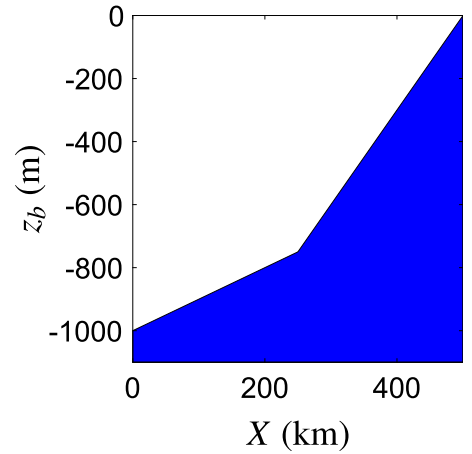

FIG. 5. Comparison of the basal melt rates obtained from (28) (solid lines) and the full plume model (dashed lines) for different idealized ice-shelf geometries with varying slope and uniform ambient properties $\left(S_{a}=34.65 \mathrm{psu}\right.$, $T_{a}=-1.9^{\circ} \mathrm{C}$ ). (left) Unscaled results. (center) Scaled plume model result compared with the dimensionless melt-rate curve $\mathscr{C}_{0}(\tilde{x})$. (right) Draft of the used geometries: (a)-(c) convex, (d)-(f) concave, (g)-(i) periodically varying slope, and (j)-(l) abrupt change in slope. 
(a)



(b)

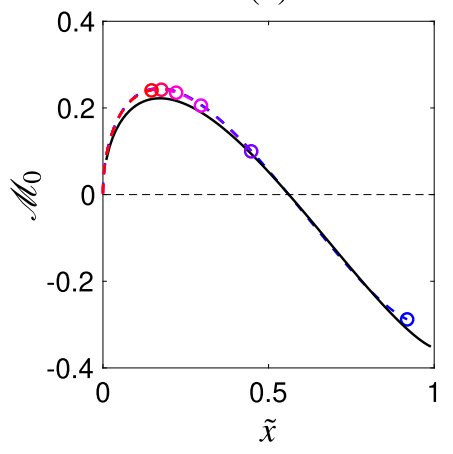

(c)

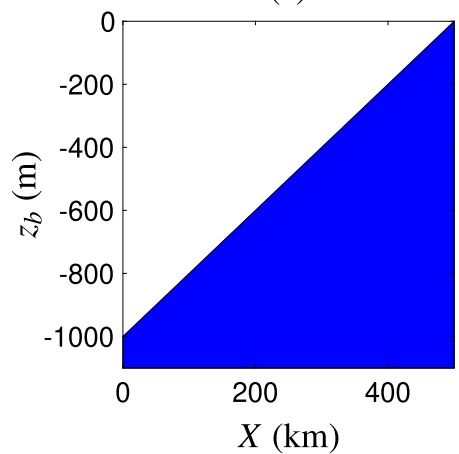

$T_{a}:-1.9{ }^{\circ} \mathrm{C}--1.1{ }^{\circ} \mathrm{C}--0.3{ }^{\circ} \mathrm{C}-+0.5^{\circ} \mathrm{C}-+1.3{ }^{\circ} \mathrm{C}-+2.1{ }^{\circ} \mathrm{C}$

FIG. 6. Comparison of the basal melt rates obtained from (28) (solid lines) and the full plume model (dashed lines) for varying but uniform ambient temperature $T_{a}$, uniform salinity $S_{a}=34.65 \mathrm{psu}$, and a fixed ice-shelf geometry with constant slope. (left) Unscaled results. (center) Scaled plume model result compared with the dimensionless melt-rate curve $\mathscr{C}_{0}(\tilde{x})$, with circles indicating the endpoint of the plume at $X=500 \mathrm{~km}$. (right) Draft of the used geometry.

showed that a deeper grounding line leads to a higher melting peak because the freezing point $T_{f, 0}$ decreases with depth. By varying the (still uniform) ambient temperature $T_{a}$, keeping the geometry and other variables fixed, we obtain the results shown in Fig. 6 for a constant basal slope, as in Fig. 4. The results show the same qualitative behavior and good agreement between the parameterization and the plume model as in the previous section. The effect of increasing $T_{a}$ is immediately clear: the melt rates increase in the whole domain, while the position of the melting peak (in the dimensional sense) appears to shift to the right. When $T_{a}$ is sufficiently high (in this case $-1.4^{\circ} \mathrm{C}$ ), the region of refreezing disappears and melting occurs in the entire domain. The deeper reasons for this behavior are illustrated by the scaled curves in Fig. 6b. All plume model results for the different values of $T_{a}$ lie along the same curve, but increasing $T_{a}$ causes the endpoint of the plume (indicated by circles and corresponding to $X=500 \mathrm{~km}$ ) to shift to the left. Hence, the effect of increasing $T_{a}$ in the parameterization is twofold: in the dimensionless sense the range of $\tilde{x}$ decreases because a larger depth change would be required to compensate the higher thermal driving at the grounding line (causing the refreezing region to disappear), but in the dimensional sense the melt-rate scale increases quadratically according to (28a). It also follows that the position of the ice-shelf front can correspond to any value of $\tilde{x}$ between 0 and 1 depending on the ambient temperature, where $\tilde{x}=1$ can only be approached if $T_{a}$ is close to the surface freezing point (section 2d).

The preceding results confirm that the parameterization performs well compared with the plume model for different uniform values of $T_{a}$. We now briefly comment on the effect of uniform values of salinity $S_{a}$. This quantity appears in the parameterization in the first factor of (28a), as well as indirectly through the freezing point $T_{f, 0}$ and the (small) parameter $c_{\tau}$. In this sense, it determines the initial strength of buoyancy on the meltwater plume. Since absolute values of salinity vary only slightly within a given region (e.g., a few percent around 34 psu in Antarctica; see, e.g., Zweng et al. 2013), the value of $S_{a}$ used in either the parameterization or the plume model will only have a very small effect on the results, as long as it is assumed uniform. In other words, the current, unstratified formulation of the parameterization can be used in regional simulations [e.g., Lazeroms et al. (2018) for Antarctica] without a spatially varying field for $S_{a}$, as the absolute values in this field will not significantly affect the melt rates.

However, this does not hold when stratification (vertically varying $T_{a}$ and $S_{a}$ ) is taken into account. In fact, stratification might be the most important phenomenon that is absent in the derivation of section 2. In reality, stratification causes plumes to detach from the ice-shelf base when reaching levels of neutral buoyancy, leading to new plumes that are uncoupled from the groundingline conditions. These form the well-known subshelf melting modes described by Jacobs et al. (1992). As shown by Jenkins (2011), the strength of the stratification can be described by a stratification length scale $l_{\rho} \sim \Delta \rho /\left(\partial \rho_{a} / \partial z\right)$, and determining the relative importance of stratification boils down to a comparison of $l_{\rho}$ with the currently used freezing-point length scale $l_{0}$. The numerical results of Jenkins (2011) reveal an interesting difference between Antarctic ice shelves 
(a)

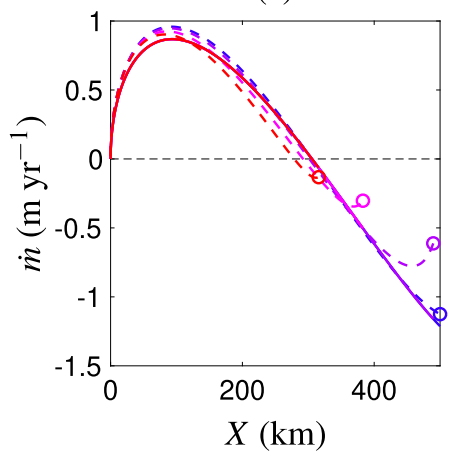

(b)

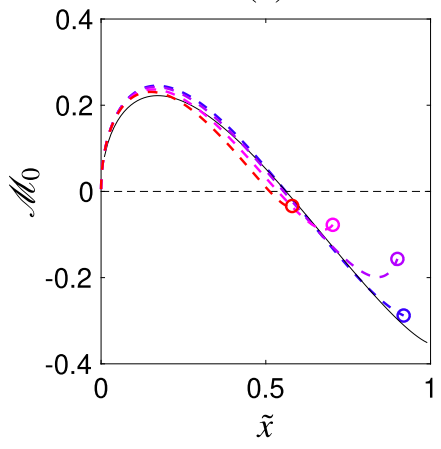

(c)

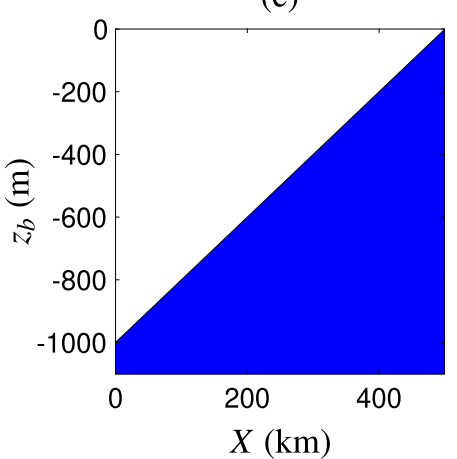

salinity gradient $\left(\mathrm{psu} \mathrm{m}^{-1}\right):-0-0.75 \times 10^{-4}-2.25 \times 10^{-4}-3.75 \times 10^{-4}$

FIG. 7. As in Fig. 6, but for nonuniform ambient salinity $S_{a}$ (stable stratification) and uniform ambient temperature $T_{a}=-1.9^{\circ} \mathrm{C}$. The circles now indicate the variable endpoint of the plume model, which is integrated until buoyancy changes sign. In all cases, the salinity equals $34.65 \mathrm{psu}$ at $X=0$ and decreases with vertical gradients shown in the legend.

$\left(l_{0} \ll l_{\rho}\right)$ and Greenland fjords $\left(l_{0} \gg l_{\rho}\right)$, indicating that stratification is typically less important for Antarctica than for Greenland.

Formally, the current formulation of both the plume model and the parameterization only describes the first melting mode from the grounding line, but it is interesting to evaluate the models for a nonuniform ambient ocean and investigate their behavior. Figure 7 shows the results of this evaluation for the same case as Fig. 6, but with uniform $T_{a}$ and vertically varying, stably stratified $S_{a}$. First, note that all curves show roughly the same behavior close to the grounding line, because the grounding-line conditions (thermal driving and slope) are equal. However, as the plume moves up toward levels of lower $S_{a}$, it eventually reaches a point of neutral buoyancy and the evaluation of the plume model is stopped before the buoyancy changes sign. These endpoints are again indicated in Fig. 7 by circles (note that the true endpoints should be at $\dot{m}=0$ when extrapolated from the numerical results).

As far as the parameterization is concerned, Fig. 7 leads to the reassuring conclusion that it remains close to the plume model in most of the evaluated domain, except in the direct vicinity of the endpoint of the plume. This can be explained by noting again that the absolute value of $S_{a}$ does not change significantly and has a negligible impact on the parameterization output. Near the endpoint, the formulation of section 2 breaks down in a way similar to the case of uniform ambient ocean (cf. Fig. 3b). Nevertheless, it appears that the essentially unstratified parameterization (28) can still be applied in stratified environments if the position of the endpoint can be identified and parameterized. A detailed discussion of such an extension is beyond the scope of this work, but estimates of the stratification length scale $l_{\rho}$ and its impact on the plume model were given by Jenkins (2011). In practical simulations, one might also consider defining different uniform layers in the ice-shelf cavity corresponding to the melting modes described by Jacobs et al. (1992) and applying the current formulation separately to each layer, similar to what was done by Magorrian and Wells (2016) for near-vertical glaciers terminating in a strongly stratified ocean.

\section{c. Realistic flow line data}

After investigating the effects of a changing slope and changing ambient temperature separately in the aforementioned idealized cases, we now turn to the evaluation of the melt-rate parameterization for more realistic geometries. Figure 8 compares the results from the plume model and the parameterization for three different ice-shelf geometries based on flow line data of FRIS (Bombosch and Jenkins 1995), Ross ice shelf (Shabtaie and Bentley 1987), and Pine Island Glacier (PIG; Crabtree and Doake 1982). These results should be compared with those shown in Lazeroms et al. (2018) for the same flow lines of FRIS and Ross ice shelf using the qualitatively similar parameterization of Jenkins (2014), as discussed in section 2d.

Note that all cases shown in Fig. 8 have uniform ambient ocean properties $T_{a}$ and $S_{a}$. Although it is technically possible to use depth-dependent profiles for these quantities, such data is usually only available from observations near the ice-shelf front and not within the ice-shelf cavity, especially for the larger ice shelves. Therefore, it is unclear if using such observed profiles for $T_{a}$ and $S_{a}$ would give more realistic results. The lack of ocean data within the cavities and the associated modeling issues were discussed extensively in 
Lazeroms et al. (2018). In that study, a two-dimensional effective ocean temperature field was constructed by inverse modeling, causing the modeled basal melt rates to match area-averaged values from the observations by Rignot et al. (2013). Hence, we use this constructed field to obtain characteristic values for $T_{a}$ for each ice shelf, yielding $T_{a}=-1.9^{\circ} \mathrm{C}$ for FRIS and Ross and $T_{a}=-1.0^{\circ} \mathrm{C}$ for PIG. Furthermore, we take $S_{a}=34.65 \mathrm{psu}$ as in the previous sections. It is important to stress that these ocean temperatures are only effective values that yield a plausible order of magnitude for the basal melt within the current modeling framework, as many more details of the cavity circulation (e.g., stratification, discussed in section 3b) and the bathymetry are required to realistically model $T_{a}$ and $S_{a}$ and the associated melt-rate profiles.

The results in Fig. 8 essentially combine the effects of a varying slope shown in Fig. 5 into a much more complicated profile. For both FRIS and Ross, the parameterized melt rates closely agree with the results of the full plume model. In particular, the FRIS profile shows a transition from melting to refreezing which is again perfectly predicted. The Ross profile remains within the positive melt region and shows a slightly better agreement between the plume model and the parameterization, mostly due to the smaller slopes in the ice-shelf base. On the other hand, the PIG profile shows a considerable discrepancy between $X \approx 15 \mathrm{~km}$ and $X \approx 40 \mathrm{~km}$, partly caused by the very steep basal slope at $X \approx 15 \mathrm{~km}$ (cf. the gray curve in Fig. 4) and partly by the rather abrupt change in the slope, giving an abrupt change in the parameterized melt rate as opposed to the more gradual behavior of the plume model (cf. Figs. 5j-1). However, the parameterized melt rate appears to "catch up" with the plume model further along the path and the overall order of magnitude remains comparable.

To indicate how the modeled melt rates in Fig. 8 relate to observations for these realistic ice shelves, we show the averaged melt rates along the flow lines in Table 2, comparing again the values obtained with the full plume model and the analytical expression. Clearly, both models capture low melt rates for Ross, slightly higher melt rates for FRIS, and relatively high melt rates for PIG, at least for the chosen values of $T_{a}$. The same trend can be seen in the area-averaged data of Rignot et al. (2013), although it is problematic to give a direct comparison of these area averages with the line averages presented here. Furthermore, we have assumed a monotonically increasing iceshelf base along the flow line, which is not always valid for realistic geometries. For a more thorough discussion on applying the parameterization to realistic $3 \mathrm{D}$ ice shelf geometries, see Lazeroms et al. (2018).
Finally, we briefly comment how the region of influence of the neglected Coriolis forces can be estimated. As discussed by Jenkins (2011), the validity of the plume equations (1) is limited by the Ekman number rather than the Rossby number, that is, friction remains more important than rotation as long as the plume thickness is smaller than the Ekman length. This was estimated by Jenkins (2011) as $D<0.24 C_{d}^{1 / 2} U /[f \cos (\alpha)]$. In this inequality, we can now substitute the analytical solutions for $\mathscr{D}$ and $\mathscr{C}$ (appendix A), which leads to

$$
\left[1-(1-\tilde{x})^{4 / 3}\right]^{1 / 2}(1-\tilde{x})^{-2 / 3}<0.34 \frac{C_{d}^{1 / 2}}{f \cos (\alpha)} \frac{\sigma_{U}(\eta)}{\sigma_{D}}
$$

where $\sigma_{U}$ and $\sigma_{D}$ are the dimensional scales of $U$ and $D$ as determined from (10) and (19). Note that $\sigma_{U}$ still depends on the slope parameter $\eta$ while the dependence on $\tau$ disappears from the ratio $\sigma_{U} / \sigma_{D}$. A quick analysis of this inequality shows that for the typical values of $\eta$ given in Fig. 2 and for $f=10^{-4} \mathrm{~s}^{-1}$, the maximum value of $\tilde{x}$ for which the inequality holds ranges from 0.2 (smaller slopes) to 0.6 (higher slopes). Comparing this to Fig. 8, we can conclude that rotational effects likely become important for larger shelves such as FRIS and Ross, but less important for smaller shelves such as PIG. However, close to the grounding line up to $\tilde{x} \approx 0.2$ there is a general region in which the Coriolis effect might be neglected.

\section{Conclusions}

We provided a systematic derivation of the basal melt rate $\dot{m}$ as a function of the scaled distance from the grounding line $\tilde{x}$ as obtained from the plume model of Jenkins (1991) for constant basal slope and uniform ambient ocean properties, using the simplified twoequation model of McPhee (1992) for the ice-ocean interface conditions. Mathematically, the derivation consists of an asymptotic analysis of the governing plume equations in the limit of small basal slope and small initial buoyancy. The resulting equation (28) for the basal melt rate consists of a melt-rate scale depending on basal slope $\alpha$ and thermal driving $\tau=T_{a}-T_{f, 0}$ and multiplied by a dimensionless melt-rate function $\mathscr{L}_{0}(\tilde{x})$, which is given by the compact expression in (26). The vertical distance from the grounding line is scaled by the length scale $\tau / \lambda_{3}$, governing the plume dynamics through the pressure dependence of the freezing point and associated thermal driving, and multiplied by a slope-dependent factor that incorporates some first-order effects, leading to the coordinate $\tilde{x}$ given by (28b). The dimensionless function $\mathscr{L}_{0}$ replaces the empirically derived polynomial curve found by Jenkins (2014) and applied to all Antarctic ice shelves in Lazeroms et al. (2018). 
(a)

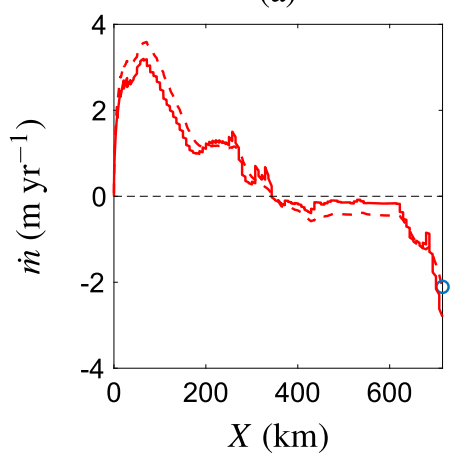

(d)

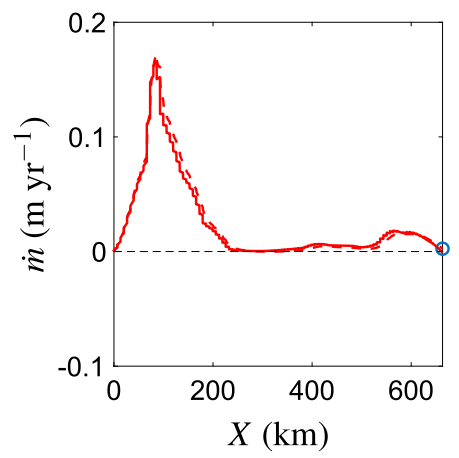

(g)

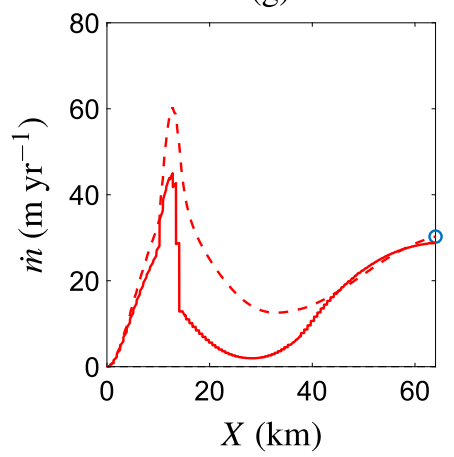

(b)

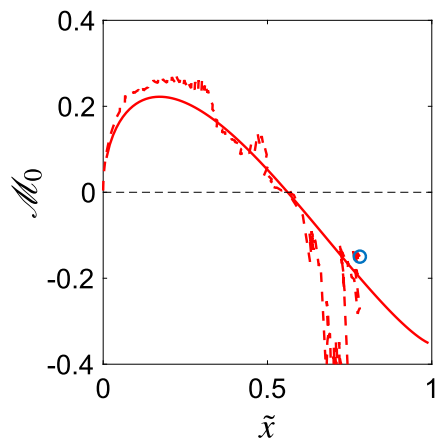

(e)

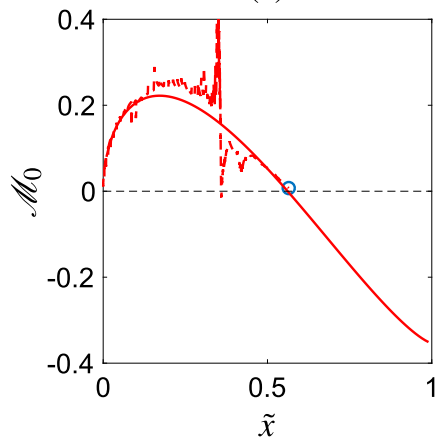

(h)

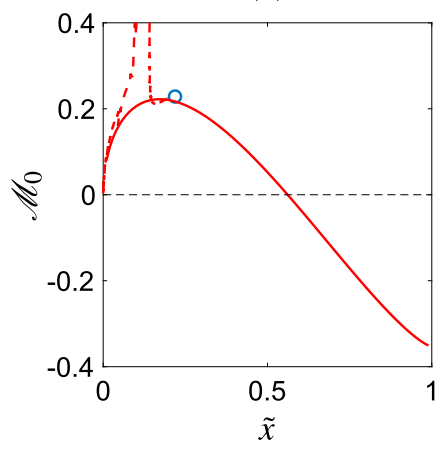

(c)

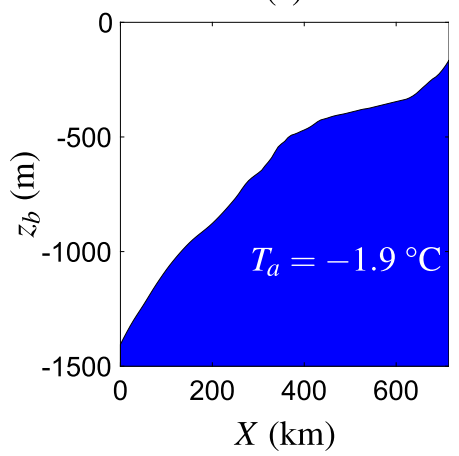

(f)

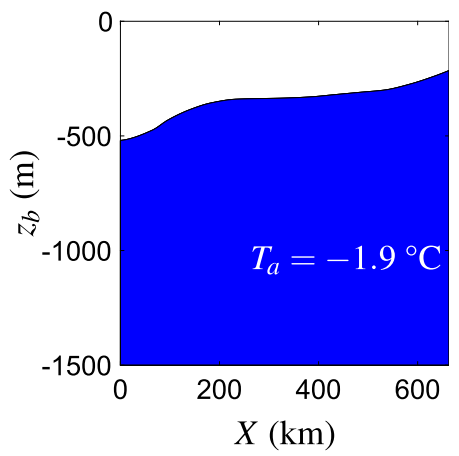

(i)

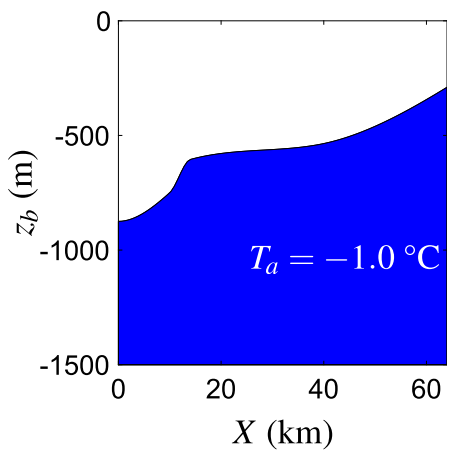

FIG. 8. As in Fig. 5, but for three realistic ice-shelf geometries along the flow lines of (a)-(c) FRIS, (d)-(f) Ross ice shelf, and (g)-(i) PIG. Note that we used a different value for the ambient ocean temperature beneath PIG $\left(T_{a}=-1.0^{\circ} \mathrm{C}\right)$, deemed characteristic for the warmer waters in the Amundsen Sea. Circles indicate the endpoint of the plume.

The advantage of the current derivation is twofold. First, it provides more insight into the main processes governing the plume dynamics and the resulting basal melt rate. The assumptions made to obtain the dimensionless function $\mathscr{L}_{0}$ are arguably close to the minimal requirements for the desired behavior in Fig. 4, showing a positive melting peak close to the grounding line and a possible transition to refreezing further down the plume path. As shown in Lazeroms et al. (2018), this behavior is needed to obtain more realistic melt-rate patterns compared with simpler parameterizations currently used in ice-sheet models.
Second, the expression for $\mathscr{C}_{0}$ in (26) is compact and easier to implement in ice-sheet models than the original empirical curve of Jenkins (2014) and Lazeroms et al. (2018). The original curve is expressed as a polynomial with 11 coefficients, whose 16-digit values should be accurately copied to avoid an incorrect implementation. The current formulation in terms of (26) would be recommended for a more robust implementation. It should be noted, however, that both formulations are numerically very similar, as Fig. 3 shows, and both formulations should have a similar impact on practical ice-sheet model simulations when implemented correctly. 
TABLE 2. Modeled basal melt rates $\left(\mathrm{m} \mathrm{yr}^{-1}\right)$ averaged over the length of the flow lines shown in Fig. 8.

\begin{tabular}{lcc}
\hline \hline Ice shelf & Plume model & Analytical expression \\
\hline FRIS & 0.56 & 0.54 \\
Ross & 0.026 & 0.024 \\
PIG & 22.1 & 15.0 \\
\hline
\end{tabular}

Furthermore, the numerical results in section 3 show that the parameterization works well compared with the full plume model (including the three-equation model for the interface conditions) for various iceshelf geometries and ambient ocean conditions in the regime where buoyancy dominates plume dynamics, even though theoretically its derivation is only valid for highly idealized cases. The largest discrepancies are visible where the basal slope is locally large or rapidly varying, due to the fact that higher-order terms in the (constant) slope were neglected in the derivation, but overall the parameterization remains close to the plume model as the latter responds more gradually to slope changes. Technically, the current formulation also breaks down in the vicinity of the plume endpoint, caused by the decrease of buoyancy and momentum close to $\tilde{x}=1$. However, this regime will likely not be reached for most realistic ice shelf geometries, unless the ambient temperature $T_{a}$ is close to the surface freezing point (explained in section $2 \mathrm{~d}$ and shown in Fig. 6) or the plume detaches from the ice shelf due to stratification (Fig. 7). One should also note that the original plume model (1) might be less valid here due to the neglected Coriolis forces and along-stream variations in buoyancy, as discussed in sections $2 \mathrm{a}$ and $3 \mathrm{c}$.

In the case of stratified ambient water, an extension of the current model to multiple uniform layers (Magorrian and Wells 2016) might be necessary to account for detached plumes and different melting modes, as briefly noted in section $3 \mathrm{~b}$. Such an extension can be formulated in terms of a stratification length scale $l_{\rho}$, as discussed by Jenkins (2011) and in section 3b. For strongly stratified regimes, $l_{\rho}$ takes over the role of $l_{0}$ as the dominant scale determining the plume extent, an effect clearly shown in Fig. 7. Another important process not present in the current formulation is the effect of tidal currents. As shown by, for example, Mueller et al. (2012), tidal forcing produces high melt rates near the calving front of ice shelves in regions where the current model typically yields refreezing. The absence of this effect can also be seen in the results of Lazeroms et al. (2018) for the larger ice shelves when comparing these to observational data (e.g., Rignot et al. 2013). Finally, we mention the subglacial discharge of meltwater at the grounding line extensively discussed by Jenkins (2011), which can significantly impact the melt rates in the immediate vicinity of the grounding line. Jenkins (2011) provided a similar parameterization for this regime, but also showed that for typical ice shelves the associated length scale is much smaller than the length scale $l_{0}$ considered here.

From a practical viewpoint, the current study only focuses on the quasi-one-dimensional plume dynamics along a single ice-shelf flow line with uniform ambient ocean properties. Other aspects need to be considered before the derived parameterization can be applied to realistic three-dimensional ice-shelf geometries for use in ice-sheet models. The two most important issues were discussed extensively in Lazeroms et al. (2018), namely the extension of the quasi-1D setting to $2 \mathrm{D}$ shelves and the required oceanic forcing field. For the first issue, Lazeroms et al. (2018) proposed a practical solution in the form of an algorithm that searches for multiple plume paths in each ice-shelf point and taking average, effective values for both the grounding-line depth $z_{\mathrm{gl}}$ and the basal slope $\alpha$ in order to calculate the basal melt rate in that point. Of course, this algorithm is not unique and it is still uncertain how sensitive the computed melt rates are to the method used to find effective plume paths.

The second issue of finding a suitable ocean forcing field might be more problematic, as observational data within ice-shelf cavities are sparse. For this reason, Lazeroms et al. (2018) constructed an effective temperature field from extrapolated ocean data by constraining the modeled basal melt rates to present-day observations from Rignot et al. (2013). The resulting forcing field contains horizontal variations in the ocean temperature (e.g., relatively warmer waters in the Amundsen Sea as in Figs. 8g-i), but it lacks information about seasonal variability and vertical profiles. Hence, the proper way to model the oceanic forcing needs to be investigated further. As a next step, horizontal variations in the ocean conditions could be incorporated by defining different coastal sectors, each with its own effective temperature and salinity. Vertical variations and stratification could then be included by using the multiple uniform layers mentioned previously. It should be pointed out that the method of constructing an effective temperature by inversion of the melt rates not only corrects for unknown temperature data, but also intrinsic biases in the melt-rate parameterization itself. The resulting temperature field should therefore be interpreted with care.

All in all, the current derivation of the basal melt parameterization is an important step in improving the description of ice-ocean interaction in ice-sheet models without fully resolving the ice-shelf cavity circulations. Its relatively simple formulation contains the minimal amount of physics needed to obtain the spatial variations in the 
melt rate between the grounding line and the ice-shelf front that cannot be captured by simpler models.

Acknowledgments. Financial support for W.M.J. Lazeroms was provided by the Netherlands Organisation for Scientific Research (NWO-ALW-Open 824.14.003). Financial support for A. Jenkins was provided by the Natural Environment Research Council, Grant NE/N01801X/1. The first author wishes to acknowledge the hospitality of the WDY Group at the Department of Applied Physics, Eindhoven University of Technology, where part of the work was done. We gratefully acknowledge the constructive comments of three anonymous reviewers.

\section{APPENDIX A}

\section{Solution in Terms of the Volume Flux $\varphi$}

Here we discuss how the dimensionless system of equations (20) for $(\mathscr{D}, \mathscr{C}, \mathscr{B}, \mathscr{T})$ can be reduced to a single equation in terms of the volume flux $\varphi=\mathscr{D} \mathscr{\mathscr { C }}$. As noted in section $2 \mathrm{~d}$, this variable is chosen because it has several convenient properties, such as

$$
\frac{d \varphi}{d x}=\mathscr{W},
$$

which directly follows from (20a). Furthermore, we can combine (20b) and (20c) to express $\mathscr{T}$ in $\varphi$ :

$$
\begin{aligned}
\mathscr{T} & =\frac{1}{\mathscr{C}} \frac{d}{d x}(\mathscr{D} \mathscr{C} \mathscr{B})=\frac{1}{\mathscr{C}} \frac{d}{d x}\left(\mathscr{C}^{3}+\delta \mathscr{D} \mathscr{b ^ { 2 }} \frac{d \mathscr{C}}{d x}\right) \\
& =3 \mathscr{C} \frac{d \mathscr{C}}{d x}+\delta \frac{d \mathscr{D} \mathscr{C}}{d x} \frac{d \mathscr{C}}{d x}+\delta \mathscr{D}\left(\frac{d \mathscr{C}}{d x}\right)^{2}+\delta \mathscr{D} \mathscr{\mathscr { C }} \frac{d^{2} \mathscr{C b}}{d x^{2}} \\
& =(3+\delta) \varphi^{\prime} \varphi^{\prime \prime}+\delta \frac{\varphi\left(\varphi^{\prime \prime}\right)^{2}}{\varphi^{\prime}}+\delta \varphi \varphi^{\prime \prime \prime} \\
& =3 \varphi^{\prime} \varphi^{\prime \prime}+O(\delta),
\end{aligned}
$$

where we have used primes to denote the $x$ derivatives of $\varphi$. It should be clear now that the assumption $\delta<O(\varepsilon)$ made in section $2 \mathrm{c}$ is necessary to make the problem manageable and avoid complicated terms with higherorder derivatives of $\varphi$. Note that we are neglecting the term $\varphi\left(\varphi^{\prime \prime}\right)^{2} / \varphi^{\prime}$, which might appear problematic for $\varphi^{\prime} \rightarrow 0$, but as we will see this singularity drops out whenever we multiply (4) with $\varphi^{\prime}$ and in particular at $x=0$ where we will obtain $\varphi \sim x^{3 / 2}$. Also note that (A1) and (A2) already give us the required expression for the dimensionless melt rate $\mathscr{C}$ in terms of $\varphi$ :

$$
\mathscr{M b}=\mathscr{Q} 6 \cdot \mathscr{T}=3\left(\varphi^{\prime}\right)^{2} \varphi^{\prime \prime}+O(\delta) .
$$

The next step is to derive a single equation for $\varphi$ and construct a solution. Equations (20c) and (20d) can be combined in the following way:

$$
\begin{aligned}
\frac{d}{d x}(\varphi \mathscr{B}) & =\frac{d}{d x}(\mathscr{D} \mathscr{C} \mathscr{R})=\mathscr{W} \mathscr{T} \\
& =(1-x) \mathscr{C}-\mathscr{D} \mathscr{C}-\varepsilon \mathscr{D} \mathscr{C} \frac{d \mathscr{T}}{d x} \\
& =(1-x) \varphi^{\prime}-\varphi\left(1+\varepsilon \frac{d \mathscr{T}}{d x}\right) \\
& =\frac{d}{d x}[\varphi(1-x-\varepsilon \mathscr{T})]+\varepsilon \mathscr{T} \varphi^{\prime} \\
& =\frac{d}{d x}[\varphi(1-x-\varepsilon \mathscr{T})]+3 \varepsilon\left(\varphi^{\prime}\right)^{2} \varphi^{\prime \prime}+O(\varepsilon \delta),
\end{aligned}
$$

where we have inserted (A2) in the last line. This equation can be directly integrated using the initial condition $\mathscr{D}=\mathscr{C}=0$ at $x=0$, that is, $\varphi(0)=\varphi^{\prime}(0)=0$ :

$$
\varphi(1-x-\varepsilon \mathscr{T}-\mathscr{B})+\varepsilon\left(\varphi^{\prime}\right)^{3}+O(\varepsilon \delta)=\text { constant }=0,
$$

which together with (A2) yields

$$
\varphi \mathscr{B}=\varphi\left(1-x-3 \varepsilon \varphi^{\prime} \varphi^{\prime \prime}\right)+\varepsilon\left(\varphi^{\prime}\right)^{3}+O(\varepsilon \delta) .
$$

Finally, (20b) yields

$$
\varphi \mathscr{B}=\mathscr{D} \mathscr{C} \mathscr{B}=\mathscr{C}^{3}+\delta \mathscr{D} \mathscr{\mathscr { C } ^ { 2 }} \frac{d \mathscr{C}}{d x}=\left(\varphi^{\prime}\right)^{3}+\delta \varphi \varphi^{\prime} \varphi^{\prime \prime},
$$

which after substitution of (A4) leads to the desired equation for $\varphi$ :

$$
(1-\varepsilon)\left(\varphi^{\prime}\right)^{3}-\varphi\left[1-x-(3 \varepsilon+\delta) \varphi^{\prime} \varphi^{\prime \prime}\right]+O(\varepsilon \delta)=0,
$$

with initial conditions $\varphi(0)=\varphi^{\prime}(0)=0$, that is, zero flux at the grounding line. It is interesting to note that these initial conditions impose an inherent singularity in the problem at $x=0$ when trying to solve for $\varphi^{\prime \prime}$ because physically there is always a small nonzero meltwater flux required to generate the plume. The current situation can be considered as the limit in which this initial flux goes to zero.

For simplicity, we can assume $\delta=O\left(\varepsilon^{2}\right)$ instead of the more general $\delta<O(\varepsilon)$ and focus on the small parameter $\varepsilon$. We can then construct an (approximate) analytical solution to (A5) by using an asymptotic expansion:

$$
\varphi(x ; \varepsilon)=\varphi_{0}(x)+\varepsilon \varphi_{1}(x)+O\left(\varepsilon^{2}\right) .
$$


The zeroth-order equation for $\varphi_{0}$ is obtained by taking the limit $\varepsilon \rightarrow 0$ in (A5):

$$
\left(\varphi_{0}^{\prime}\right)^{3}=(1-x) \varphi_{0}, \quad \varphi_{0}(0)=0,
$$

again reducing the order of the system but with the condition $\varphi_{0}^{\prime}(0)=0$ implied by the equation. Equation (A7) can be solved analytically by standard methods. Formally, we can obtain both positive and negative realvalued solutions of (A7), as well as the trivial solution $\varphi_{0}(x)=0$. The occurrence of these different branches can be understood by considering that (A7) holds in the limit $\varepsilon \rightarrow 0$, hence the limit of zero slope, in which there is no longer a distinction between upward and downward moving plumes. Hence, an additional causality condition $\varphi_{0}(x)>0$ for $0<x<1$ is required to obtain the true physical solution.

For $0 \leq x \leq 1$, the positive real-valued solution of (A7) has the following form:

$$
\varphi_{0}(x)=\frac{1}{2 \sqrt{2}}\left[1-(1-x)^{4 / 3}\right]^{3 / 2} .
$$

From $\varphi_{0}$ we can directly obtain the expression for the leading-order velocity:

$$
\mathscr{C}_{0}(x)=\varphi_{0}^{\prime}(x)=\frac{1}{\sqrt{2}}(1-x)^{1 / 3}\left[1-(1-x)^{4 / 3}\right]^{1 / 2}
$$

Furthermore, the leading-order plume thickness, density difference, temperature difference and melt rate are given by

$$
\begin{aligned}
& \mathscr{D}_{0}(x)=\varphi_{0} \mathscr{\mathscr { C }}_{0}=\frac{1}{2}(1-x)^{-1 / 3}\left[1-(1-x)^{4 / 3}\right], \quad(\mathrm{A} 8 \mathrm{c}) \\
& \mathscr{B}_{0}(x)=\mathscr{C}_{0}^{2} / \mathscr{D}_{0}=1-x, \quad(\mathrm{~A} 8 \mathrm{~d}) \\
& \mathscr{T}_{0}(x)=3 \varphi_{0}^{\prime}(x) \varphi_{0}^{\prime \prime}(x)=\frac{1}{2}\left[3(1-x)-\frac{1}{(1-x)^{1 / 3}}\right], \quad \text { and } \\
& \mathscr{C}_{0}(x)=\mathscr{C}_{0} \mathscr{T}_{0}=\frac{1}{2 \sqrt{2}}\left[3(1-x)^{4 / 3}-1\right]\left[1-(1-x)^{4 / 3}\right]^{1 / 2} .
\end{aligned}
$$

Note that, since the expression for $\mathscr{C}_{0}$ is equivalent to $d\left[\left(\varphi_{0}^{\prime}\right)^{3}\right] / d x$, the leading order balance (A7) simply expresses the integrated meltwater flux $\left(\varphi_{0}^{\prime}\right)^{3}$ as a function of the total plume volume flux $\varphi_{0}$. Their ratio $(1-x)$ is the volume fraction of meltwater within the plume, and that determines the plume properties relative to the ambient. Thus, the $(1-x)$ term appears repeatedly in the expressions for $\mathscr{C}_{0}, \mathscr{D}_{0}$, and $\mathscr{T}_{0}$, while the scaled density difference $\mathscr{B}_{0}$ is exactly equal to $(1-x)$ because it arises directly from the admixture of meltwater in the plume. Adding stratification to the model likely changes the latter expression so that it decreases to zero more quickly, modifying the $(1-x)$ terms in the other expressions accordingly. Another interesting point is that $\mathscr{C}_{0}$ includes $(1-x)^{1 / 3}$, which is the analog of the standard plume scaling (Jenkins 2011) in which the velocity scales as the buoyancy flux to the third power.

Figure A1 shows $\varphi_{0}, \mathscr{C}_{0}, \mathscr{D}_{0}, \mathscr{R}_{0}, \mathscr{T}_{0}$, and $\mathscr{C}_{0}$ as functions of $x$. These curves have several interesting properties. At $x=0$, we have $\varphi_{0}=\mathscr{D}_{0}=\mathscr{C}_{0}=\mathscr{C}_{0}=0$, as desired, while $\mathscr{B}_{0}=\mathscr{T}_{0}=1$. Note that this also means that $\varphi^{\prime \prime}$ and all higher derivatives are singular at $x=0$, since $\varphi^{\prime}=0$ but $3 \varphi^{\prime} \varphi^{\prime \prime}=1$. This singularity is caused by the fractional exponents present in (A8). In particular, by applying the relation $(1-x)^{n} \approx 1-n x$ to (A8a), we obtain the asymptotic relations [up to $O(1)$ prefactors] $\varphi \sim x^{3 / 2}, \varphi^{\prime} \sim x^{1 / 2}$, and $\varphi^{\prime \prime} \sim x^{-1 / 2}$, revealing the singularity in $\varphi^{\prime \prime}$ at $x=0$. A similar singularity is present at $x=1$, where $\varphi^{\prime}$ again goes to 0 and $\varphi^{\prime \prime}$ to $-\infty$, but here the product of $\varphi^{\prime}$ and $\varphi^{\prime \prime}$ is not sufficient to cancel out the singularity, causing $\mathscr{T}_{0}$ to go to $-\infty$ as well. On the other hand, the melt rate $\mathscr{A b}_{0}$ is well behaved at $x=1$, as the singularity is cancelled in the product of $\left(\varphi^{\prime}\right)^{2}$ and $\varphi^{\prime \prime}$, causing $\mathscr{C l}_{0}$ to go to a finite value here.

To summarize, we have found a zeroth-order approximation of the solution of (A5) and, as a direct consequence, a closed expression for the melt-rate curve. This solution is only valid for $0 \leq x \leq 1$ and contains inherent singularities at $x=0$ and $x=1$ (although it remains possible to construct a second real-valued branch for $x>1$, this solution will not be physical because it represents negative velocities). Near $x=0$, the curves provide a close approximation to the full solution (Fig. 3a), with the desired behavior of the melt rate: a positive peak near $x=0.2$ and transition to refreezing further on. The biggest discrepancy occurs around $x=1$, where the zeroth-order solution becomes singular, whereas the full solution continues beyond $x=1$ depending on the value of $\varepsilon$. Finally, note that the approximate solution can potentially be improved by including the first-order term $\varphi_{1}$ and using $\varphi \approx \varphi_{0}+\varepsilon \varphi_{1}$, which also depends on $\varepsilon$. The expression for $\varphi_{1}$ is

$$
\begin{aligned}
\varphi_{1}(x)= & -\frac{1}{24 \sqrt{2}}\left[1-(1-x)^{4 / 3}\right]^{1 / 2} \times\left[3-3(1-x)^{1 / 3}\right. \\
& \left.+3 x(1-x)^{1 / 3}+4 \log (1-x)\right],
\end{aligned}
$$

which was found by substituting $\varphi \approx \varphi_{0}+\varepsilon \varphi_{1}$ in (A5), collecting the $O(\varepsilon)$ terms, and solving the resulting differential equation for $\varphi_{1}$ using computer algebra software. It turns out that by using $\varphi_{1}$, the discrepancy 
between the approximate solution and the full solution indicated in Fig. 3a is improved in a large part of the domain, including the position of the transition point. However, the inherent singularity at $x=1$ remains in the form of the logarithmic term in (A9), and no higher-order approximation is able to cancel it. Physically, the singularity at $x=1$ can be traced back to neglecting the temperature gradient [see (20d) with $\varepsilon \rightarrow 0$ ]. A possible way to get rid of the singularity might be a boundary layer around $x=1$ where the temperature gradient is taken into account at leading order. This is discussed in appendix B.

\section{APPENDIX B}

\section{Boundary Layer Solution around $x=1$}

In perturbation theory, boundary layers typically occur in higher-order problems with multiple boundary conditions, for which an asymptotic expansion [e.g., (16)] found by standard methods turns out to satisfy only a subset of these boundary conditions (see, e.g., Mattheij et al. 2005). The most well-known example of such behavior is fluid flow close to a solid wall or object, where a boundary layer region close to the surface is required to adapt the essentially inviscid outer flow to the viscous boundary conditions at the surface. In other words, a straightforward asymptotic expansion in the limit of small viscosity will fail close to the surface because viscosity becomes dominant here.

A similar situation occurs in our asymptotic approximation of (20) and (23): in the limit of small $\varepsilon$, we are essentially neglecting the temperature gradient in (20d), but this terms turns out to be dominant in a region around $x=1$, where our approximation appears to fail (see also Fig. A1), not only in the zeroth-order term but in higher-order terms as well. A possible solution might be to consider a second asymptotic expansion in the region around $x=1$ (the boundary layer) where the temperature gradient is not neglected.

In this case, a boundary layer solution can be found by rescaling the distance as follows:

$$
x=1+\varepsilon^{m} \xi,
$$

where $\varepsilon^{m}$ is the (still unknown) boundary layer thickness and $\xi$ can have either positive or negative values. A first guess of the behavior of the solution can be found by substituting the rescaled $x$ in (25) and expanding it in a Taylor series around $\varepsilon=0$ :

$\varphi_{0}\left(1+\varepsilon^{m} \xi\right)=\frac{1}{2 \sqrt{2}}-\frac{3(-\xi)^{4 / 3}}{4 \sqrt{2}} \varepsilon^{4 m / 3}+O\left(\varepsilon^{8 m / 3}\right)$,

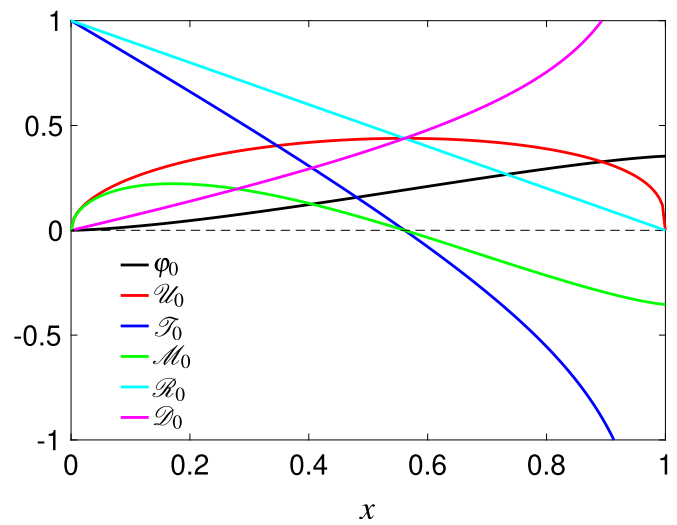

FIG. A1. Zeroth-order solutions for volume flux $\varphi_{0}$, velocity $\mathscr{C}_{0}$, temperature difference $\mathscr{T}_{0}$, melt rate $\mathscr{C}_{0}$, density difference $\mathscr{R}_{0}$, and plume thickness $\mathscr{D}_{0}$ given by (A8).

where $(-\xi)^{4 / 3}$ should be interpreted as a positive real number. We see that $\varphi_{0}$ tends to a finite value around $x=1(\xi=0)$ for $\varepsilon \rightarrow 0$, and the next term in the expansion is of order $\varepsilon^{4 m / 3}$. Hence, we can try the following ansatz for the behavior of $\varphi$ in the boundary layer:

$$
\varphi(x)=A_{0}+\varepsilon^{4 m / 3} Y_{1}(\xi),
$$

with constant $A_{0}$. This ansatz is substituted in (23) together with the rescaled $x=1+\varepsilon^{m} \xi$. After some tedious algebra and bookkeeping of the various terms, it turns out that a meaningful balance in (23) that retains the second-order derivative is achieved for $m=3 / 4$. Hence, we find a boundary layer thickness $\varepsilon^{3 / 4}$ and the new approximation involving $Y_{1}$ is simply a term of $O(\varepsilon)$. Taking $\varepsilon \rightarrow 0$ in the rescaled equation turns out to give

$$
\left(Y_{1}^{\prime}\right)^{3}=-A_{0}\left(\xi+3 Y_{1}^{\prime} Y_{1}^{\prime \prime}\right) .
$$

Comparing (B4) with (A7), we see that there is indeed an additional term $Y_{1}^{\prime} Y_{1}^{\prime \prime}$ related to the temperature difference. Through (A1)-(A3), it is straightforward to show that the leading-order velocity in the boundary layer is equal to $\varepsilon^{1 / 4} Y_{1}^{\prime}$ and the leading-order temperature difference is equal to $3 \varepsilon^{-1 / 4} Y_{1}^{\prime} Y_{1}^{\prime \prime}$. Hence, the leading-order melt rate is again the product of the two, $3\left(Y_{1}^{\prime}\right)^{2} Y_{1}^{\prime \prime}$.

In principle, one can now solve (B4) for $Y_{1}^{\prime}$. This will add an additional degree of freedom [e.g., $Y_{1}^{\prime}(0)=A_{1}$ ] because the boundary conditions of this equation are still unknown. Together with the still unknown constant $A_{0}$, this gives two degrees of freedom (a third degree of freedom is obtained when integrating $Y_{1}^{\prime}$ to find $Y_{1}$ ). In theory, suitable values of $A_{0}$ and $A_{1}$ could be found by matching the boundary layer solution with the outer solution $\varphi_{0}[(25)]$ in the overlap region. Unfortunately, (B4) has no analytical solution, so this exercise can only be done numerically. Fig. B1a shows an example of a 
(a)

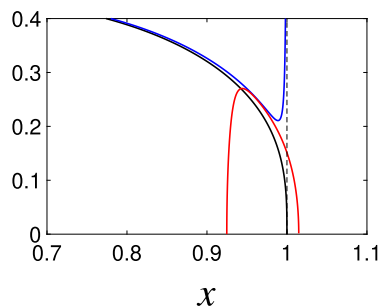

(b)

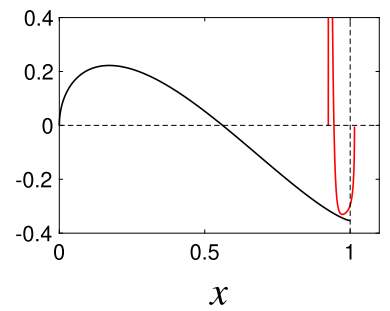

FIG. B1. (a) Matching of the boundary layer solution $\varepsilon^{1 / 4} Y_{1}^{\prime}$ (red) with the first-order outer solution for the velocity, $\varphi_{0}^{\prime}+\varepsilon \varphi_{1}^{\prime}$ (blue) for $\varepsilon=0.005$. The matching coefficients in this case are $A_{0} \approx 0.35$ and $A_{1} \approx 0.57$. The black curve is the zeroth-order solution $\varphi_{0}^{\prime}=\mathscr{\mathscr { C }} b_{0}$ shown in Fig. A1. (b) Comparison of boundary layer solution $3\left(Y_{1}^{\prime}\right)^{2} Y_{1}^{\prime \prime}$ (red) with zeroth-order melt rate $\mathscr{L}_{0}$ (black). In a region $x=1 \pm O\left(\varepsilon^{3 / 4}\right)$, the red curve describes the behavior of the melt rate near the plume endpoint, which can also be seen in Fig. 3.

numerical matching for $\varepsilon=0.005$, which also uses the derivative of $\varphi_{1}$ given by (A9). Clearly, the boundary layer solution exists in a small region around $x=1$ and causes the velocity to go to zero at a new endpoint $x=1+O\left(\varepsilon^{3 / 4}\right)$. As $\varepsilon$ goes to zero, the endpoint will move closer to $x=1$ and the solution closer to the outer solution $\varphi_{0}$. In this particular case, it appears possible to match the boundary layer solution with the first-order outer solution in an overlap region around $x=1-O\left(\varepsilon^{3 / 4}\right)$.

The previous discussion provides more theoretical insight in the solution of (23), especially around $x=1$ and in the limit $\varepsilon \rightarrow 0$. It also sheds more light on the analytical solution presented in section $2 \mathrm{~d}$ and the reasons why it is valid in a large part of the domain, except around $x=1$. Although it is theoretically possible to match the boundary layer solution with the outer solution, this does not directly provide a practical improvement for the melt-rate curve given by (26), because (B4) cannot be solved analytically. Furthermore, we explained in section $2 \mathrm{~d}$ why the region around $x=1$ has only a very limited physical meaning in practical simulations. However, we can make use of the boundary layer scaling found in the aforementioned discussion, as it follows that the endpoint of the plume scales as $x=1+O\left(\varepsilon^{3 / 4}\right)$. This suggests the ad hoc correction in (27) aimed at constraining $x$ to values between 0 and 1 , which indeed improves the agreement between the curves in Fig. 3 . Not directly captured in this way is the behavior of the melt rate in the boundary layer, where it decreases until reaching zero at the endpoint of the plume (Fig. B1b).

\section{REFERENCES}

Asay-Davis, X. S., S. L. Cornford, B. K. Galton-Fenzi, R. M. Gladstone, G. H. Gudmundsson, D. M. Holland, P. R. Holland, and D. F. Martin, 2016: Experimental design for three interrelated marine ice sheet and ocean model intercomparison projects: MISMIP v. 3 (MISMIP+), ISOMIP v. 2 (ISOMIP+) and MISOMIP v. 1 (MISOMIP1). Geosci. Model Dev., 9, 2471-2497, https://doi.org/ 10.5194/gmd-9-2471-2016.

_ , N. C. Jourdain, and Y. Nakayama, 2017: Developments in simulating and parameterizing interactions between the Southern Ocean and the Antarctic Ice sheet. Curr. Climate Change Rep., 3, 316-329, https://doi.org/10.1007/s40641-017-0071-0.

Beckmann, A., and H. Goosse, 2003: A parameterization of ice shelf-ocean interaction for climate models. Ocean Modell., 5 , 157-170, https://doi.org/10.1016/S1463-5003(02)00019-7.

Bo Pedersen, F., 1980: Dense bottom currents in rotating ocean. J. Hydraul. Div., 106, 1291-1308.

Bombosch, A., and A. Jenkins, 1995: Modeling the formation and deposition of frazil ice beneath Filchner-Ronne Ice Shelf. J. Geophys. Res., 100, 6983-6992, https://doi.org/10.1029/ 94JC03224.

Crabtree, R. D., and C. S. M. Doake, 1982: Pine Island Glacier and its drainage basin: Results from radio echo-sounding. Ann. Glaciol., 3, 65-70, https://doi.org/10.1017/S0260305500002548.

De Boer, B., and Coauthors, 2015: Simulating the Antarctic ice sheet in the Late-Pliocene warm period: PLISMIP-ANT, an ice-sheet model intercomparison project. Cryosphere, 9, 881903, https://doi.org/10.5194/tc-9-881-2015.

DeConto, R. M., and D. Pollard, 2016: Contribution of Antarctica to past and future sea-level rise. Nature, 531, 591-597, https:// doi.org/10.1038/nature17145.

Depoorter, M. A., J. L. Bamber, J. A. Griggs, J. T. M. Lenaerts, S. R. M. Ligtenberg, M. R. Van den Broeke, and G. Moholdt, 2013: Calving fluxes and basal melt rates of Antarctic ice shelves. Nature, 502, 89-92, https://doi.org/10.1038/ nature12567.

De Rydt, J., and G. H. Gudmundsson, 2016: Coupled ice shelfocean modeling and complex grounding line retreat from a seabed ridge. J. Geophys. Res. Earth Surf., 121, 865-880, https:// doi.org/10.1002/2015JF003791.

Dinniman, M. S., J. M. Klinck, L.-S. Bai, D. H. Bromwich, K. M. Hines, and D. M. Holland, 2015: The effect of atmospheric forcing resolution on delivery of ocean heat to the Antarctic floating ice shelves. J. Climate, 28, 6067-6085, https://doi.org/ 10.1175/JCLI-D-14-00374.1.

Eckhaus, W., 1979: Asymptotic Analysis of Singular Perturbations. North-Holland, $286 \mathrm{pp}$.

Golledge, N. R., D. E. Kowalewski, T. R. Naish, R. H. Levy, C. J. Fogwill, and E. G. W. Gasson, 2015: The multi-millennial Antarctic commitment to future sea-level rise. Nature, 526, 421-425, https://doi.org/10.1038/nature15706.

Hellmer, H. H., and D. J. Olbers, 1989: A two-dimensional model for the thermohaline circulation under an ice shelf. Antarct. Sci., 1, 325-336, https://doi.org/10.1017/S0954102089000490.

Holland, P. R., A. Jenkins, and D. M. Holland, 2008: The response of ice shelf basal melting to variations in ocean temperature. J. Climate, 21, 2558-2572, https://doi.org/10.1175/ 2007JCLI1909.1.

Holmes, M. H., 1995: Introduction to Perturbation Methods. Springer, $356 \mathrm{pp}$.

Jacobs, S. S., H. H. Helmer, C. S. M. Doake, A. Jenkins, and R. M. Frolich, 1992: Melting of ice shelves and the mass balance of Antarctica. J. Glaciol., 38, 375-387, https://doi.org/10.1017/ S0022143000002252.

Jenkins, A., 1991: A one-dimensional model of ice shelf-ocean interaction.J. Geophys. Res., 96, 20 671-20 677, https://doi.org/ 10.1029/91JC01842. 
2011: Convection-driven melting near the grounding lines of ice shelves and tidewater glaciers. J. Phys. Oceanogr., 41, 2279-2294, https://doi.org/10.1175/JPO-D-11-03.1.

_- 2014: Scaling laws for the melt rate and overturning circulation beneath ice shelves derived from simple plume theory. Geophysical Research Abstracts, Vol. 16, Abstract EGU2014-13755, https://meetingorganizer.copernicus.org/ EGU2014/EGU2014-13755.pdf.

Lane-Serff, G. F., 1995: On meltwater under ice shelves. J. Geophys. Res., 100, 6961-6965, https://doi.org/10.1029/94JC03244.

Lazeroms, W. M. J., A. Jenkins, G. H. Gudmundsson, and R. S. W. van de Wal, 2018: Modelling present-day basal melt rates for Antarctic ice shelves using a parametrization of buoyant meltwater plumes. Cryosphere, 12, 49-70, https://doi.org/10.5194/ tc-12-49-2018.

MacAyeal, D. R., 1985: Evolution of tidally triggered meltwater plumes below ice shelves. Oceanology of the Antarctic Continental Shelf, S. Jacobs, Ed., Antarctic Research Series, Vol. 43, Amer. Geophys. Union, 133-143.

Magorrian, S. J., and A. J. Wells, 2016: Turbulent plumes from a glacier terminus melting in a stratified ocean. J. Geophys. Res. Oceans, 121, 4670-4696, https://doi.org/10.1002/ 2015JC011160.

Mahrt, L., 1982: Momentum balance of gravity flows. J. Atmos. Sci., 39, 2701-2711, https://doi.org/10.1175/1520-0469(1982)039<2701: MBOGF $>2.0 . \mathrm{CO} ; 2$

Mathiot, P., A. Jenkins, C. Harris, and G. Madec, 2017: Explicit and parametrised representation of under ice shelf seas in a $z^{*}$ coordinate ocean model NEMO 3.6. Geosci. Model Dev., 10, 2849-2874, https://doi.org/10.5194/gmd-10-2849-2017.

Mattheij, R. M. M., S. W. Rienstra, and J. H. M. ten Thije Boonkkamp, 2005: Partial Differential Equations: Modeling, Analysis, Computation. Society for Industrial and Applied Mathematics, $665 \mathrm{pp}$.

McPhee, M. G., 1992: Turbulent heat flux in the upper ocean under sea ice. J. Geophys. Res. Oceans, 97, 5365-5379, https://doi.org/ 10.1029/92JC00239.

—, C. Kottmeier, and J. H. Morison, 1999: Ocean heat flux in the central Weddell sea during winter. J. Phys. Oceanogr., 29, 1166-1179, https://doi.org/10.1175/1520-0485(1999)029<1166: OHFITC $>2.0 . \mathrm{CO} ; 2$.

Mueller, R. D., L. Padman, M. S. Dinniman, S. Y. Erofeeva, H. A. Fricker, and M. A. King, 2012: Impact of tide-topography interactions on basal melting of Larsen C Ice Shelf, Antarctica. J. Geophys. Res., 117, C05005, https://doi.org/ 10.1029/2011JC007263.

Naughten, K. A., K. J. Meissner, B. K. Galton-Fenzi, M. H. England, R. Timmermann, H. H. Hellmer, T. Hattermann, and J. B. Debernard, 2018: Intercomparison of Antarctic iceshelf, ocean, and sea-ice interactions simulated by MetROMSiceshelf and FESOM 1.4. Geosci. Model Dev., 11, 1257-1292, https://doi.org/10.5194/gmd-11-1257-2018.

Nayfeh, A. H., 1973: Perturbation Methods. John Wiley and Sons, $425 \mathrm{pp}$.
Olbers, D., and H. Hellmer, 2010: A box model of circulation and melting in ice shelf caverns. Ocean Dyn., 60, 141-153, https:// doi.org/10.1007/s10236-009-0252-z.

Pritchard, H. D., S. R. M. Ligtenberg, H. A. Fricker, D. G. Vaughan, M. R. Van den Broeke, and L. Padman, 2012: Antarctic ice-sheet loss driven by basal melting of ice shelves. Nature, 484, 502-505, https://doi.org/10.1038/nature10968.

Reese, R., T. Albrecht, M. Mengel, X. Asay-Davis, and R. Winkelmann, 2018: Antarctic sub-shelf melt rates via PICO. Cryosphere, 12, 1969-1985, https://doi.org/10.5194/tc-12-1969-2018.

Rignot, E., S. Jacobs, J. Mouginot, and B. Scheuchl, 2013: Ice-shelf melting around Antarctica. Science, 341, 266-270, https:// doi.org/10.1126/science.1235798.

, J. Mouginot, M. Morlighem, H. Seroussi, and B. Scheuchl, 2014: Widespread, rapid grounding line retreat of Pine Island, Thwaites, Smith, and Kohler glaciers, West Antarctica, from 1992 to 2011. Geophys. Res. Lett., 41, 3502-3509, https:// doi.org/10.1002/2014GL060140.

Sergienko, O. V., 2013: Basal channels on ice shelves. J. Geophys. Res. Earth Surf., 118, 1342-1355, https://doi.org/10.1002/ jgrf.20105.

Seroussi, H., Y. Nakayama, E. Larour, D. Menemenlis, M. Morlighem, E. Rignot, and A. Khazendar, 2017: Continued retreat of Thwaites Glacier, West Antarctica, controlled by bed topography and ocean circulation. Geophys. Res. Lett., 44, 6191-6199, https://doi.org/10.1002/2017GL072910.

Shabtaie, S., and C. R. Bentley, 1987: West Antarctic ice streams draining into the Ross Ice Shelf: configuration and mass balance. J. Geophys. Res., 92, 1311-1336, https://doi.org/10.1029/ JB092iB02p01311.

Slater, D., P. Nienow, A. Sole, T. Cowton, R. Mottram, P. Langen, and D. Mair, 2017: Spatially distributed runoff at the grounding line of a large Greenlandic tidewater glacier inferred from plume modelling. J. Glaciol., 63, 309-323, https:// doi.org/10.1017/jog.2016.139.

Smedsrud, L. H., and A. Jenkins, 2004: Frazil ice formation in an ice shelf water plume. J. Geophys. Res., 109, C03025, https:// doi.org/10.1029/2003JC001851.

Thoma, M., J. Determann, K. Grosfeld, S. Goeller, and H. H. Hellmer, 2015: Future sea-level rise due to projected ocean warming beneath the Filchner Ronne Ice Shelf: A coupled model study. Earth Planet. Sci. Lett., 431, 217-224, https:// doi.org/10.1016/j.epsl.2015.09.013.

Timmermann, R., and H. H. Hellmer, 2013: Southern Ocean warming and increased ice shelf basal melting in the twentyfirst and twenty-second centuries based on coupled ice-ocean finite-element modelling. Ocean Dyn., 63, 1011-1026, https:// doi.org/10.1007/s10236-013-0642-0.

and S. Goeller, 2017: Response to Filchner-Ronne Ice Shelf cavity warming in a coupled ocean-ice sheet model-Part 1: The ocean perspective. Ocean Sci., 13, 765-776, https:// doi.org/10.5194/os-13-765-2017.

Zweng, M. M., and Coauthors, 2013: Salinity. Vol. 2, World Ocean Atlas 2013, NOAA Atlas NESDIS 74, 39 pp. 